Historic, archived document

Do not assume content reflects current scientific knowledge, policies, or practices. 

$a \leqslant 011$
.452

United States

Department of

Agriculture

Forest

Service

General

Technical

Report WO-33

\section{Tree Biomass - A State-of-the-Art Compilation}

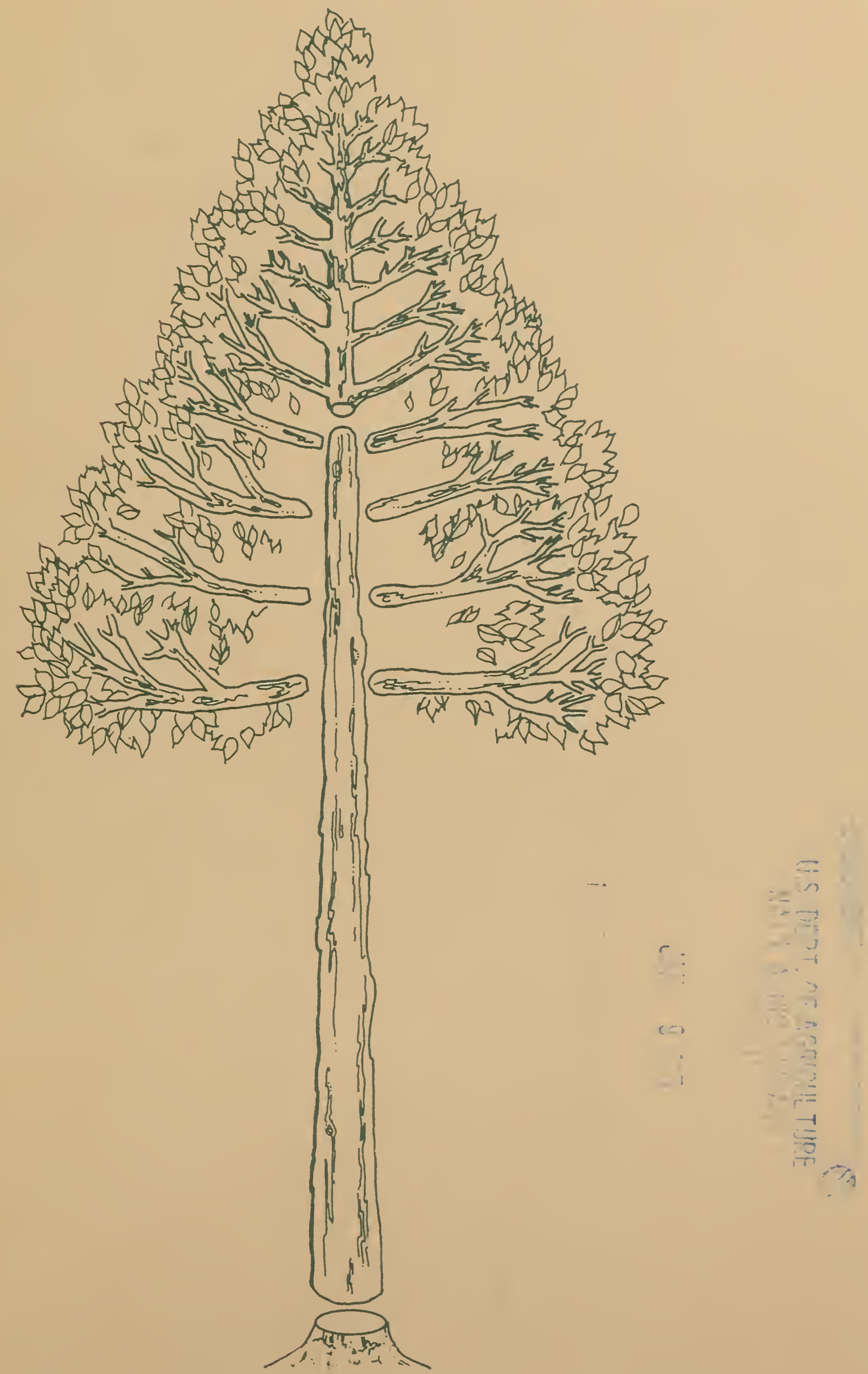



TREE BIOMASS-

A State-of-the-Art Compilation

United States Department of Agriculture

Forest Service

Washington, D.C.

General Technical Report WO-33

November 1981 


\section{National Tree Biomass Compilation Committee Members}

James $T$. Bones, National Coordinator, Washington, D.C.

Gerhard K. Raile, North Central Forest Experiment Station, St. Paul, Minn.

Eric H. Wharton, Northeastern Forest Experiment Station, Broomall, $\mathrm{Pa}$.

Noel D. Cost, Southeastern Forest Experiment Station, Asheville, N.C.

Charles E. Thomas, Southern Forest Experiment Station, New Orleans, La.
Kenneth Skog, Forest Products Laboratory, Madison, Wisc.

Dwane D. Van Hooser, Intermountain Forest and Range Experiment Station, Ogden, Utah

James 0. Howard, Pacific Northwest Forest and Range Experiment Station, Portland, Oreg.

Vernon J. LaBau, Pacific Northwest Forest and Range Experiment Station, Anchorage, Alaska 
Until recentiy, forest products were made principally from the main stem, called the merchantable bole, I/ of trees. Decreasing tree sizes, increasing extraction and processing costs, new product development, increasing competition for wood, and the energy crisis have a 11 focused attention on extending timber utilization to include the total aboveground portion of the tree. Tree crowns can be utilized as fuel for domestic and industrial purposes or, fiber for pulp and paper and composite board products, as a soil amendment or mulch in agriculture, and a bulking agent in municipal sludge composting. In view of this, whole-tree utilization is being recognized as a potentially viable forestry practice.

Because technology has preceded research programs in forest mensuration and inventory methods, information is lacking regarding the amount of wood and bark contained in the tree as a whole and particularly in the crown component. Such information is vital if land managers and timber resource planners are to be able to make logical decisions about timber management and timber procurement on a whole-tree basis. Statewide, regional, and national tree biomass statistics must be available for public and private planning purposes.

In January 1980, a National Tree Biomass Compilation Committee was formed with representatives from Renewable Resources Evaluation Research work units throughout the Nation. The committee was charged with developing a program to

I/ Terms defined in Glossary. satisfy the information needs. A three-phased program was developed.

The first phase consisted of carrying out a biomass compilation based on state-of-the-art methods that were available to the individual research work units.

In the Lake States, Rocky Mountain, and Pacific Coast regions compilation was based on converting merchantable bole volumes to weight and applying a series of conversion factors that were developed from experience data to compensate for the bark, top, and branch components. Eastern and Alaska research units were able to assemble enough usable existing tree weight equations to adopt an individual tree weight estimation approach. These tree weight equations were applied to stand tables from the most recent statewide forest inventories to produce the required weight statistics. The results of this compilation are presented in this publication.

Phase two is an ongoing process and consists of identification of research needs based on deficiencies recognized during the tree biomass compilation phase. In the west, this includes the development and improvement of tree weight equations for important tree species in locations where there are none. In the East, methods of sample tree selection for use in constructing biomass tables specifically for regional forest inventories have been identified as shortcomings. Many of the existing equations are too site or location specific, are based on too few sample trees, or sample trees are not representative of the total population of trees within species groupings. 
The third phase of the committee's work involves the integration of biomass estimation into the resources evaluation inventory system. Once this is accomplished, statewide analyses of tree biomass, its availability, and the competition between wood for fuel and for fiber products can be assessed. The time needed to realize this goal will depend to a great extent on how rapidiy the research identified in phase two can be completed and the findings applied.

\section{ACCURACY OF STATISTICS}

The statistics contained in this publication must be considered as best estimates because of the varying methods used in deriving them. While in some States the statistics are based on inventories with known sampling errors, most tree weight equations are not supported by statistically reliable tree samples. Species lacking acceptable tree weight equations were grouped with other species having similar green weight, crown form, and tree size distribution. 
1. Total green weight of aboveground tree biomass on commercial forest 1 and in the United States by class of timber, species group, section, and region.

2. Total green weight of aboveground tree biomass on commercial forest land in the North by class of timber, species group, section, and State.

3. Total green weight of aboveground tree biomass on commercial forest land in the South by class of timber, species group, section, and State.

4. Total green weight of aboveground tree biomass on commercial forest land in the West by class of timber, species group, section, and State.

5. Per-acre green weight of aboveground tree biomass on commercial forest 1 and in the North by class of timber, species group, section, and State.

6. Per-acre green weight of aboveground tree biomass on commercial forest 1 and in the South by class of timber, species group, section, and State.

7. Per-acre green weight of aboveground tree biomass on commercial forest land in the West by class of timber, species group, section, and State.

8. Total green weight of aboveground softwood tree biomass on commercial forest land in the eastern United States by species, diameter class, section, and region.

9. Total green weight of aboveground hardwood tree biomass on commercial forest land in the eastern United States by species, diameter class, section, and region.

10. Total green weight of aboveground sof twood tree biomass on commercial forest land in the western United States by species, diameter class, section, and region.

11. Total green weight of aboveground hardwood tree biomass on commercial forest land in the western United States by species, diameter class, section, and region.

12. Total green weight of aboveground tree biomass on commercial forest 1 and in the United States by ownership, section, and region.

13. Total green weight of aboveground tree biomass on commercial forest land in the North by ownership, section, and State.

14. Total green weight of aboveground tree biomass on commercial forest land in the South by ownership, section, and State.

15. Total green weight of aboveground tree biomass on commercial forest land in the West by ownership, section, and State. 


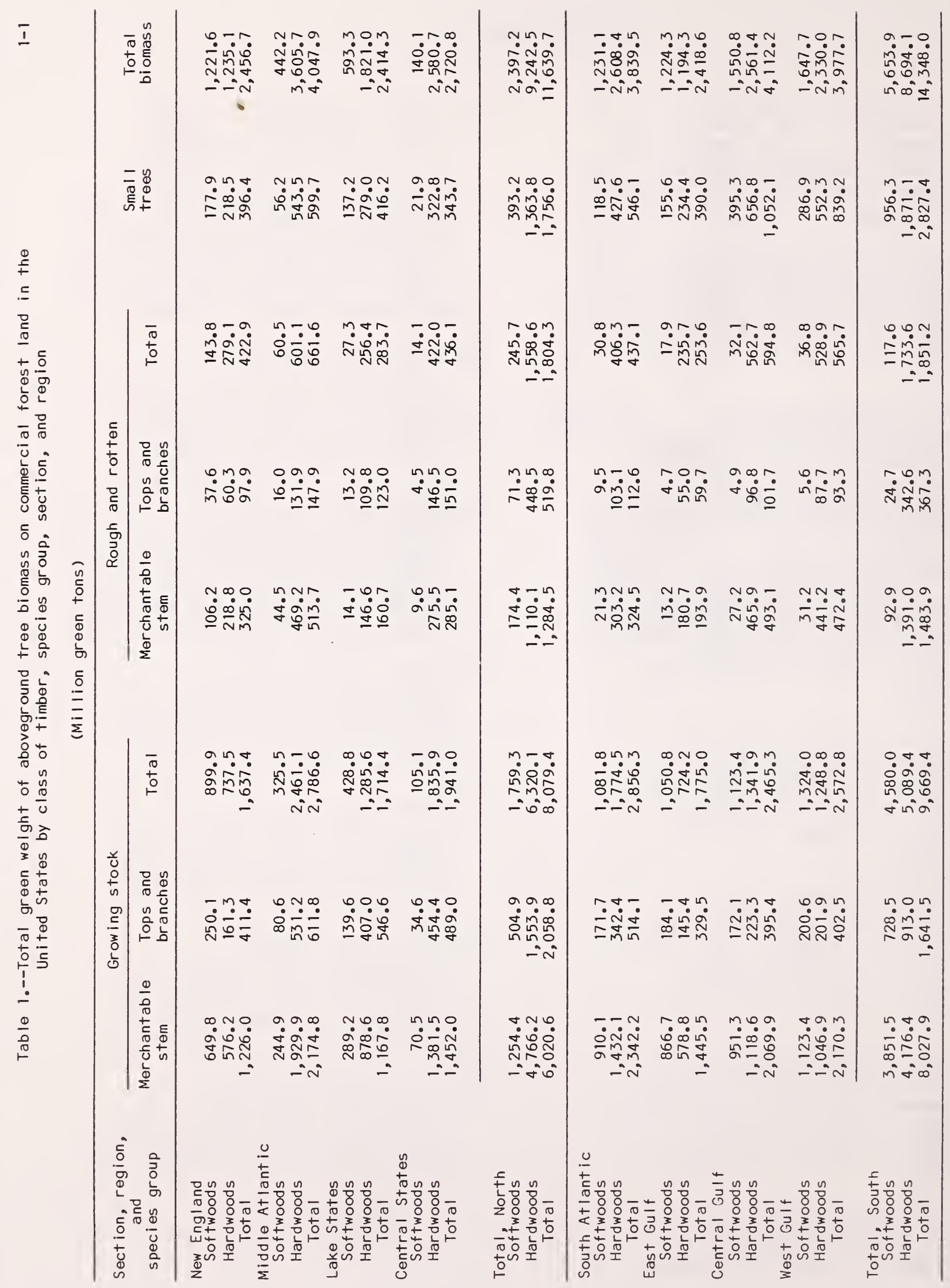




\begin{tabular}{|c|c|c|}
\hline 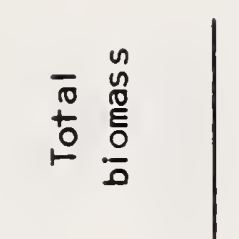 & 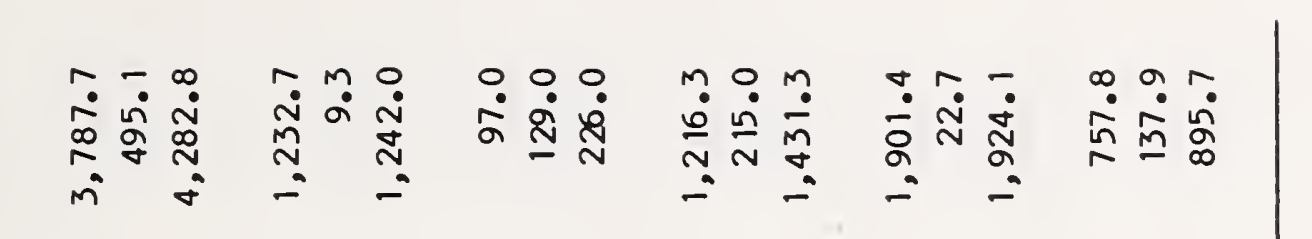 & 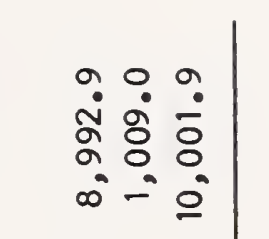 \\
\hline 率暴 & 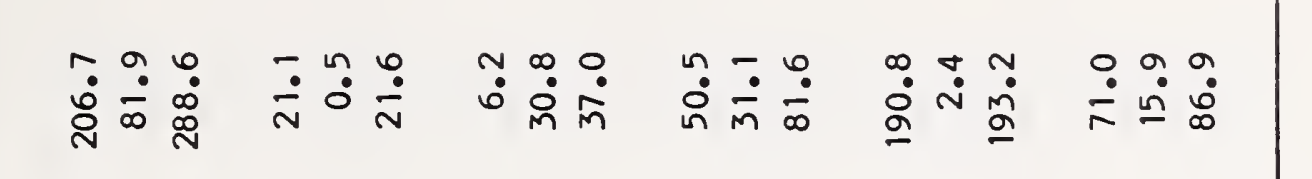 & 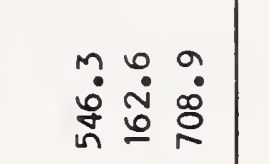 \\
\hline $\mid \frac{\bar{z}}{2}$ & 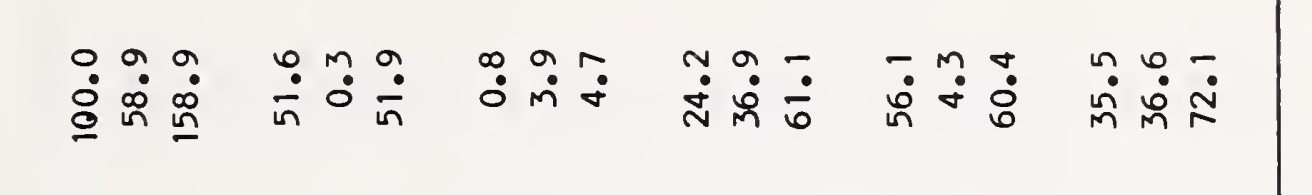 & 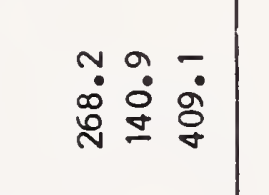 \\
\hline 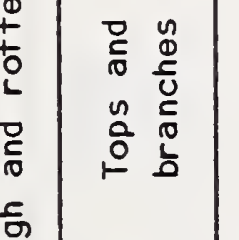 & 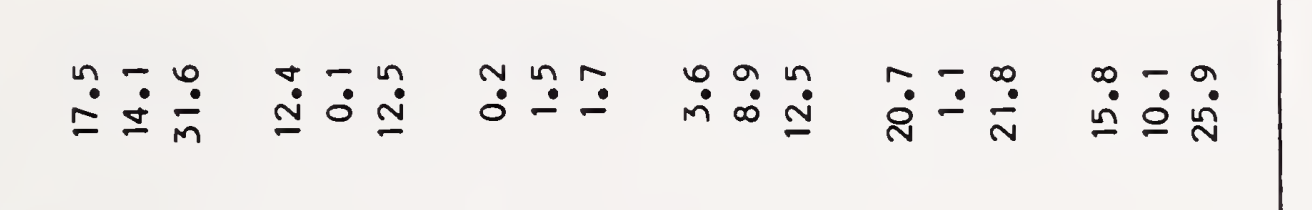 & 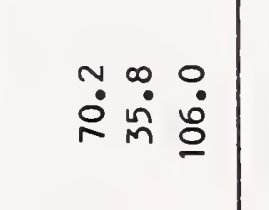 \\
\hline $\mid$ & 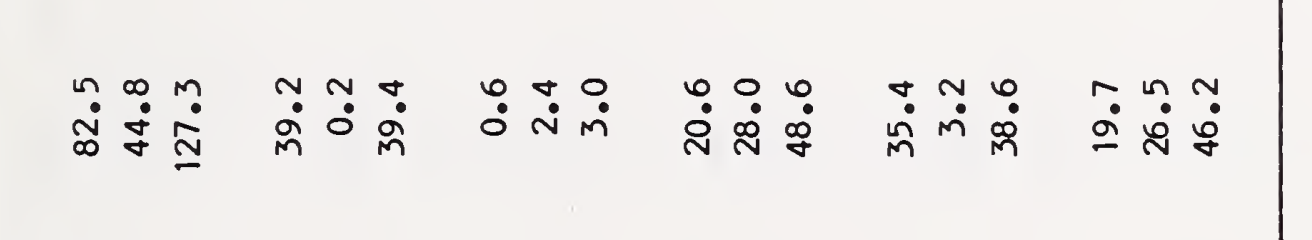 & 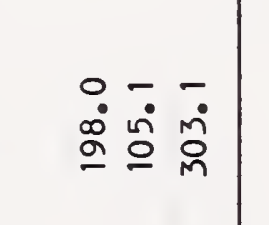 \\
\hline$\left|\frac{\bar{z}}{2}\right|$ & 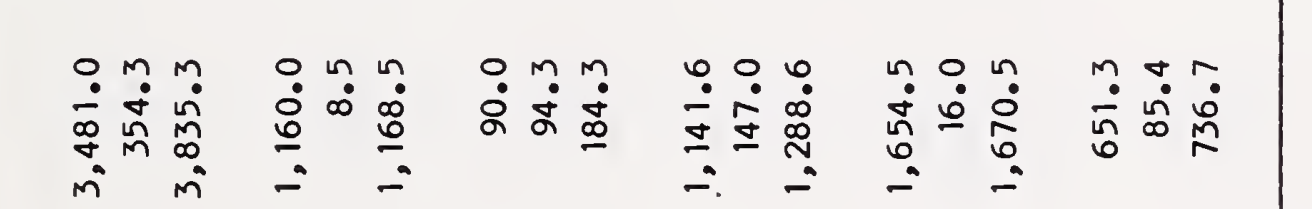 & 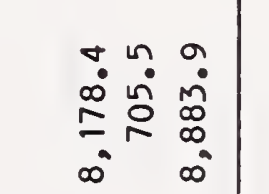 \\
\hline 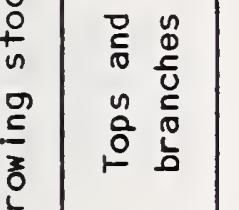 & 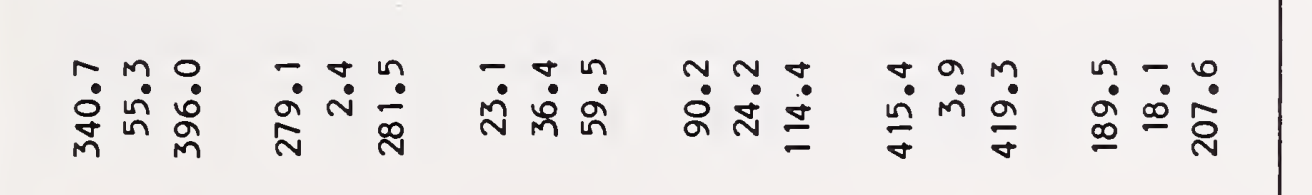 & 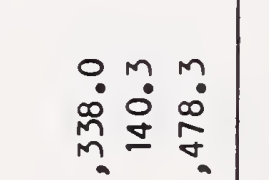 \\
\hline $\mid$ & 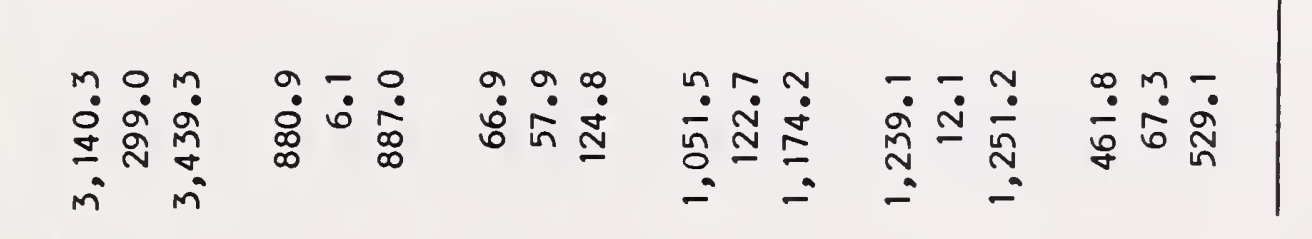 & 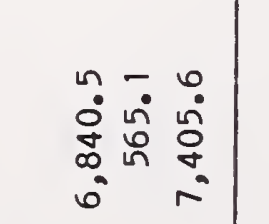 \\
\hline
\end{tabular}




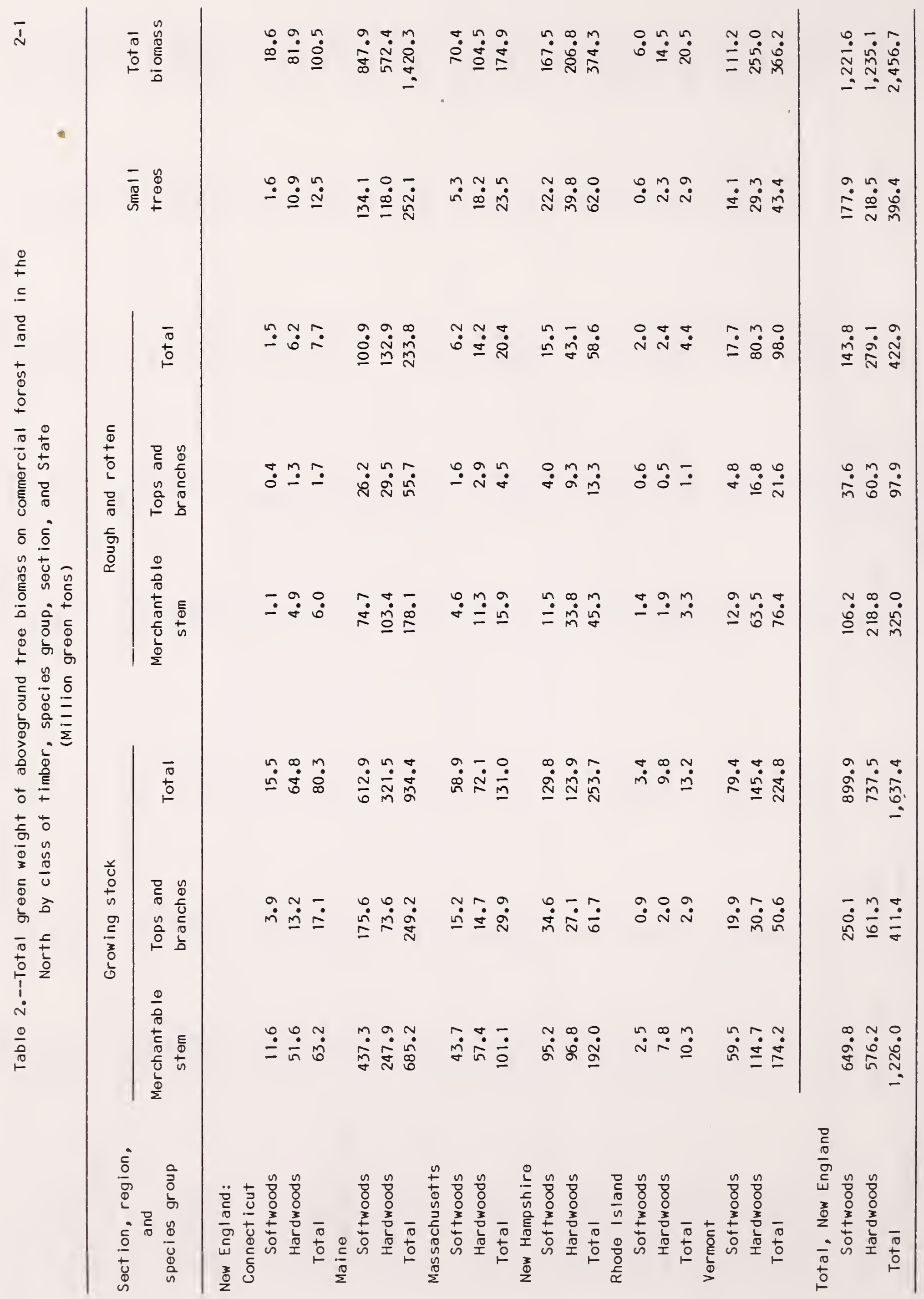




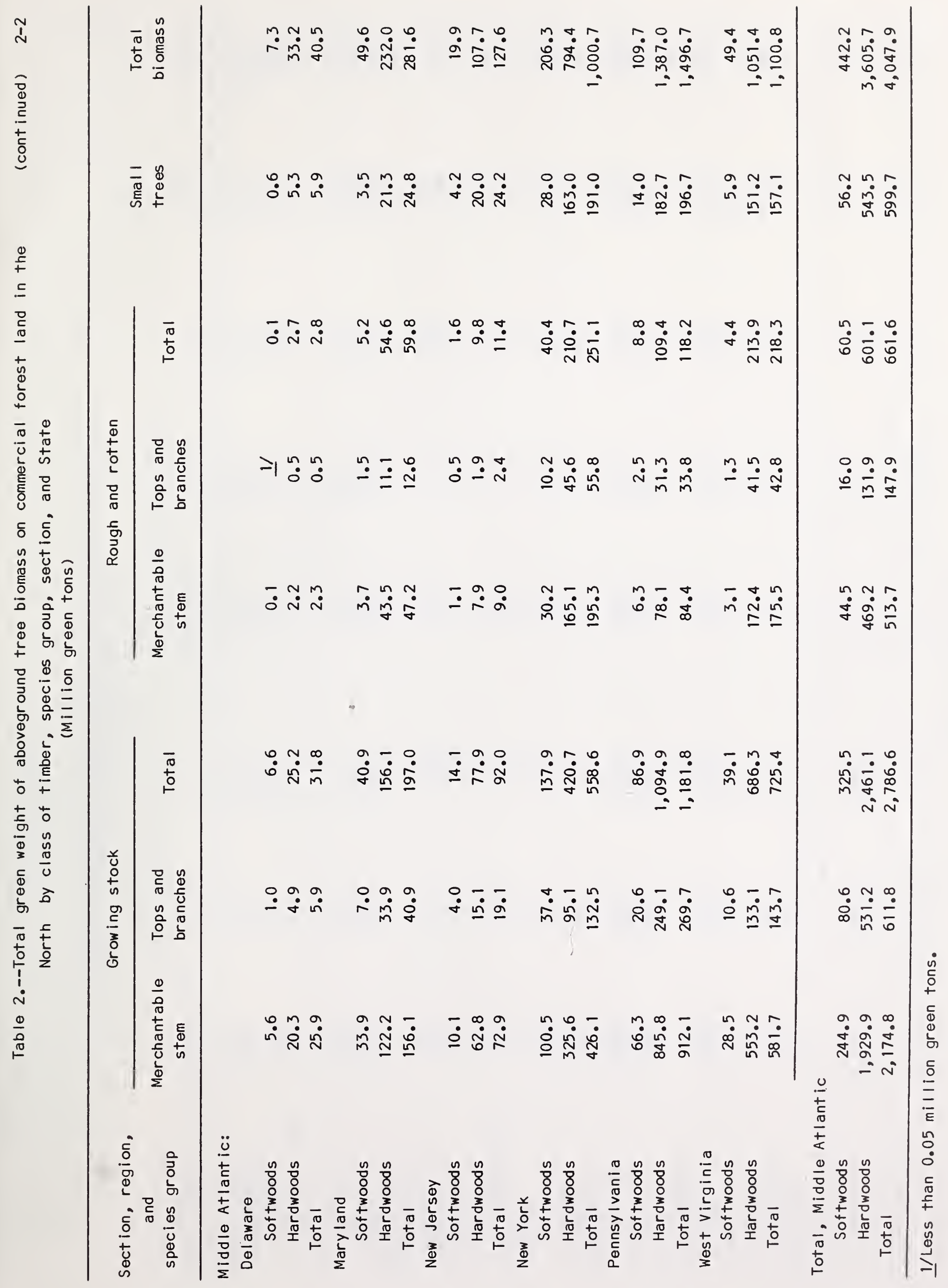




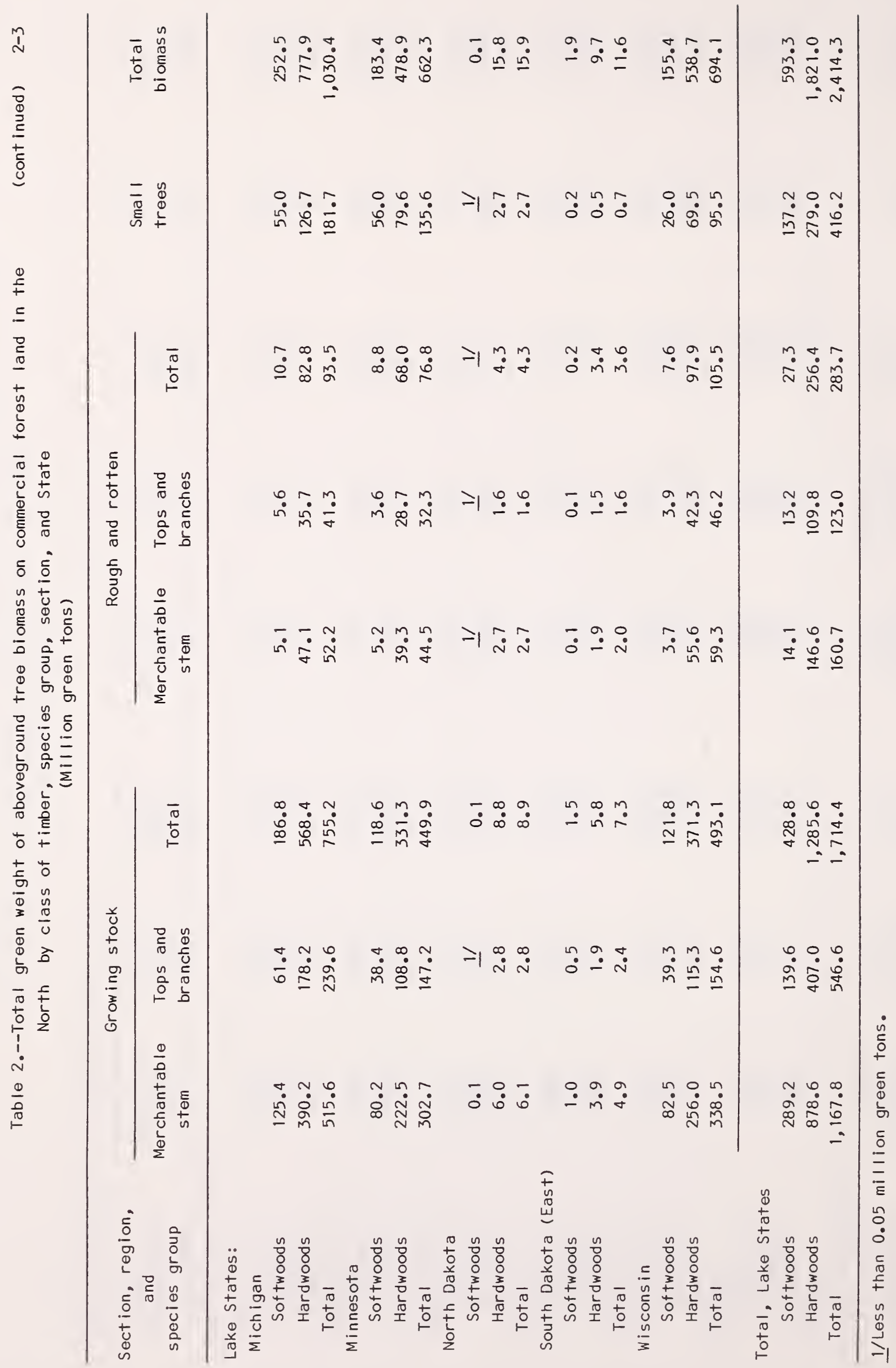




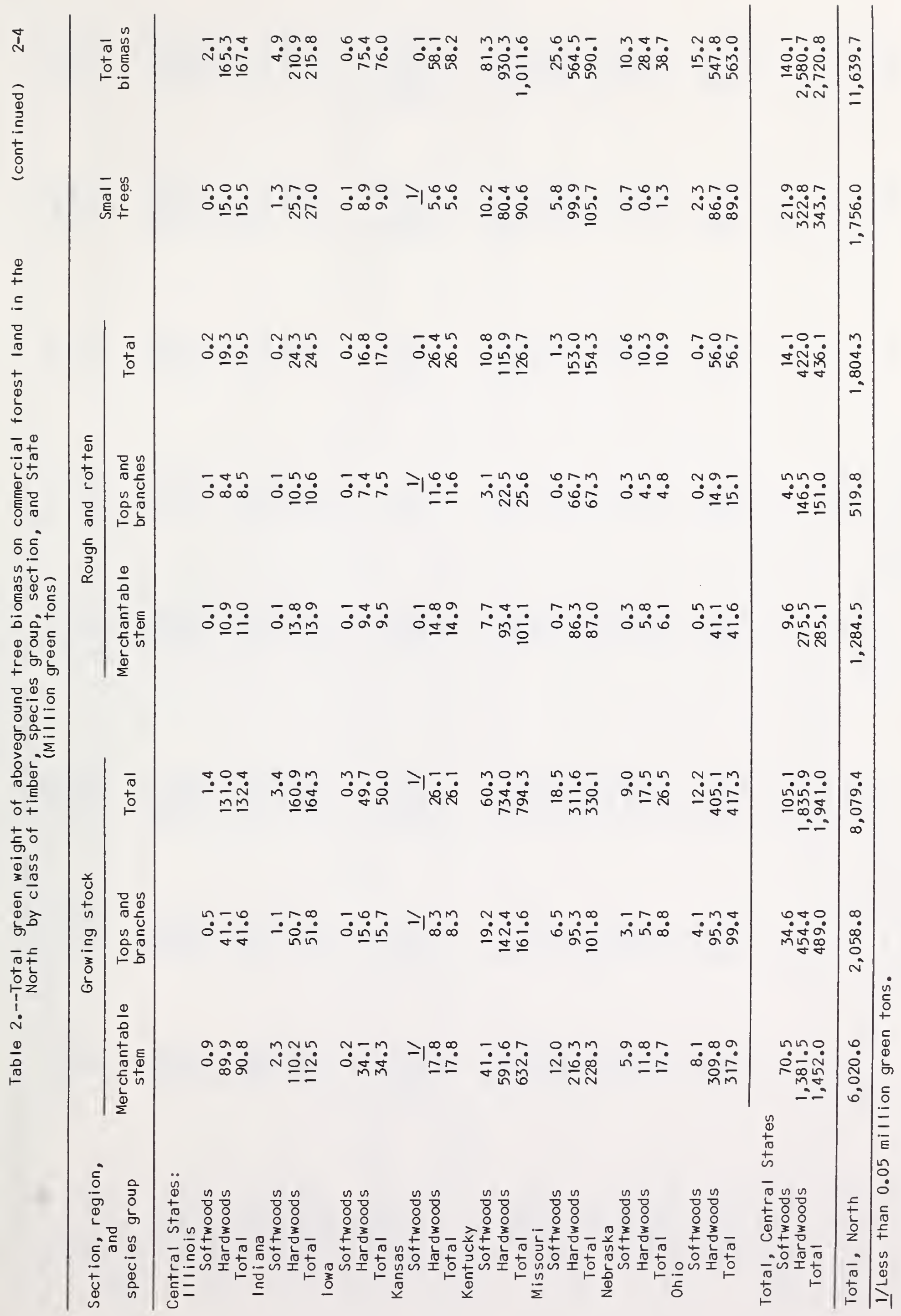




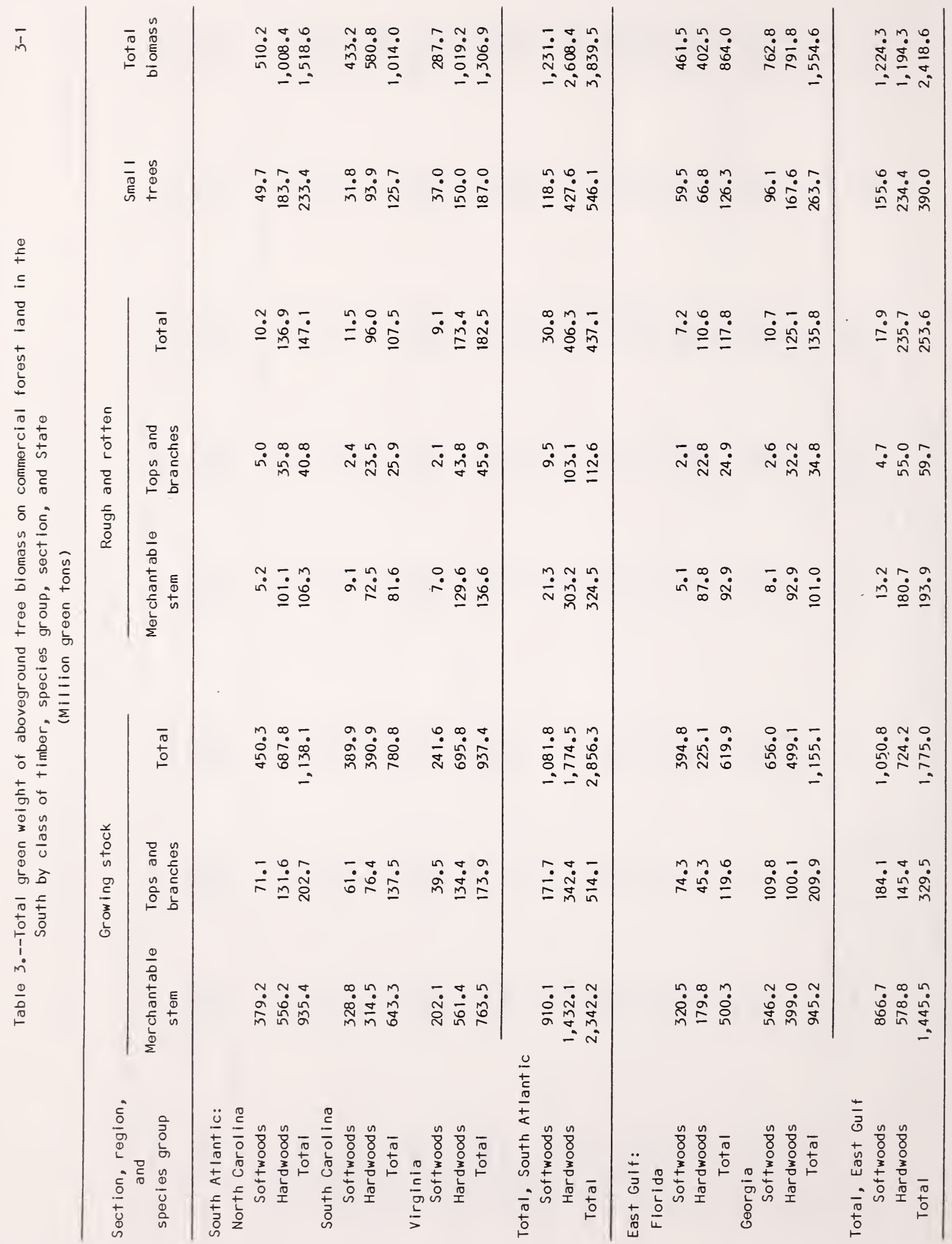




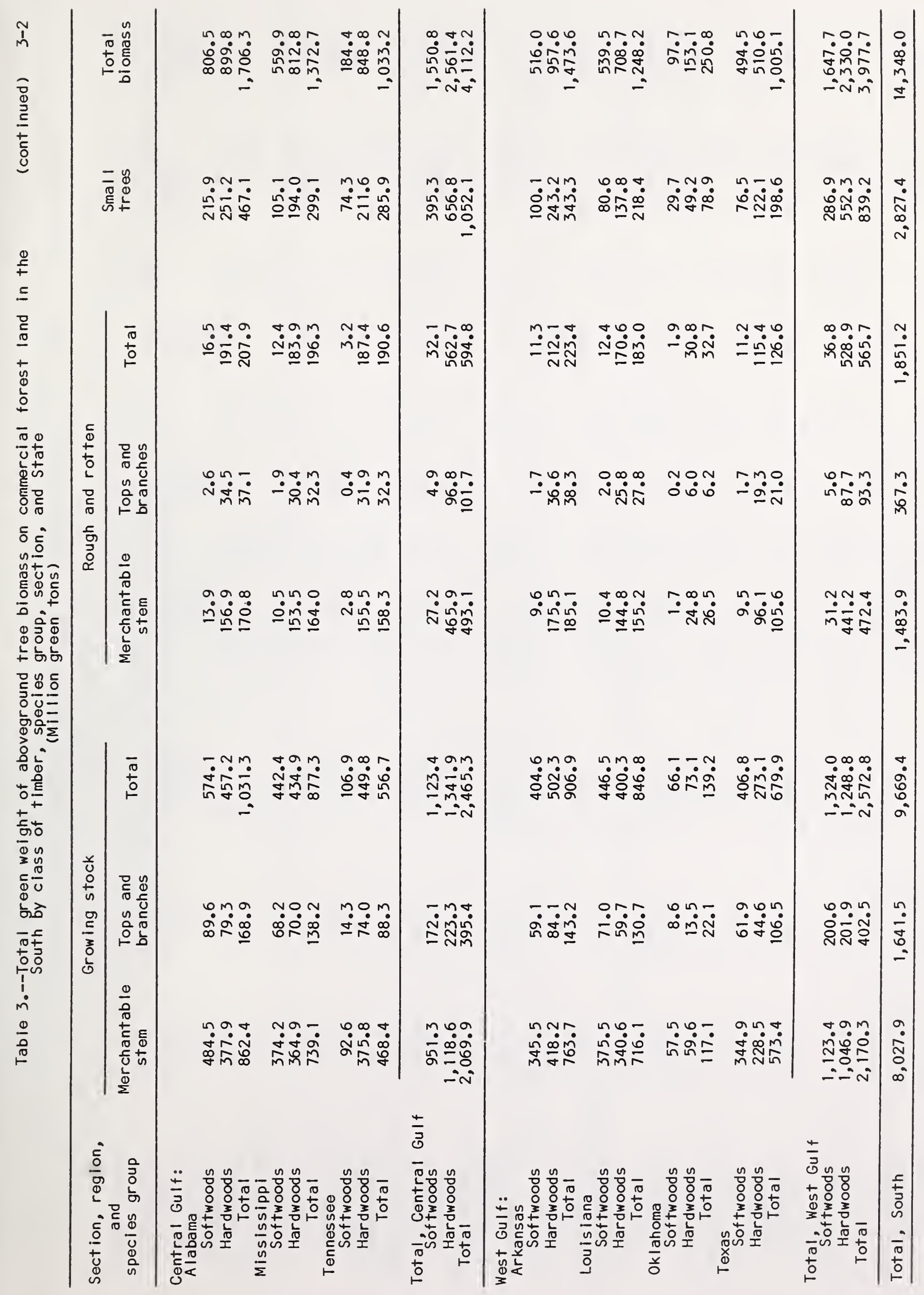




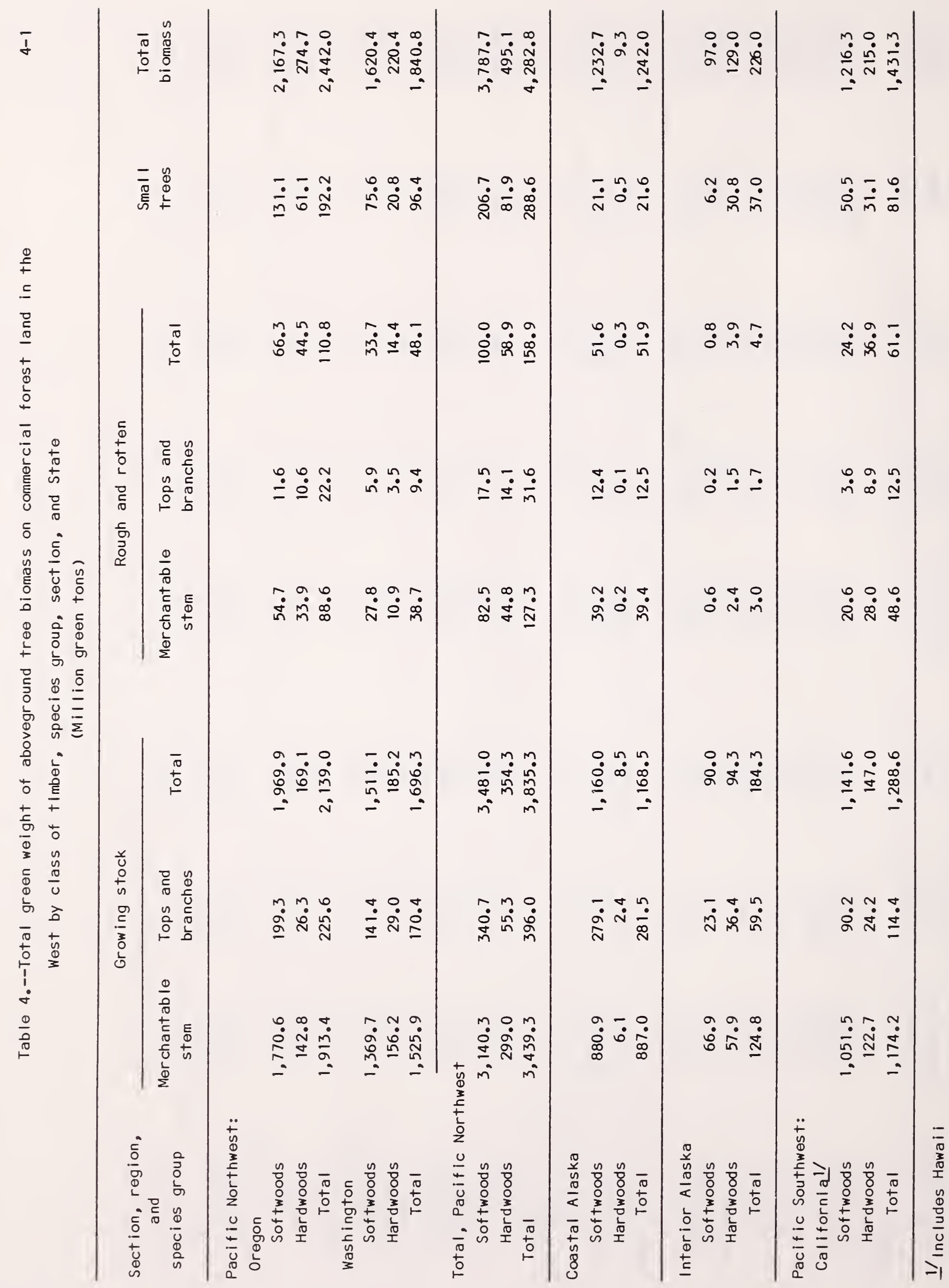




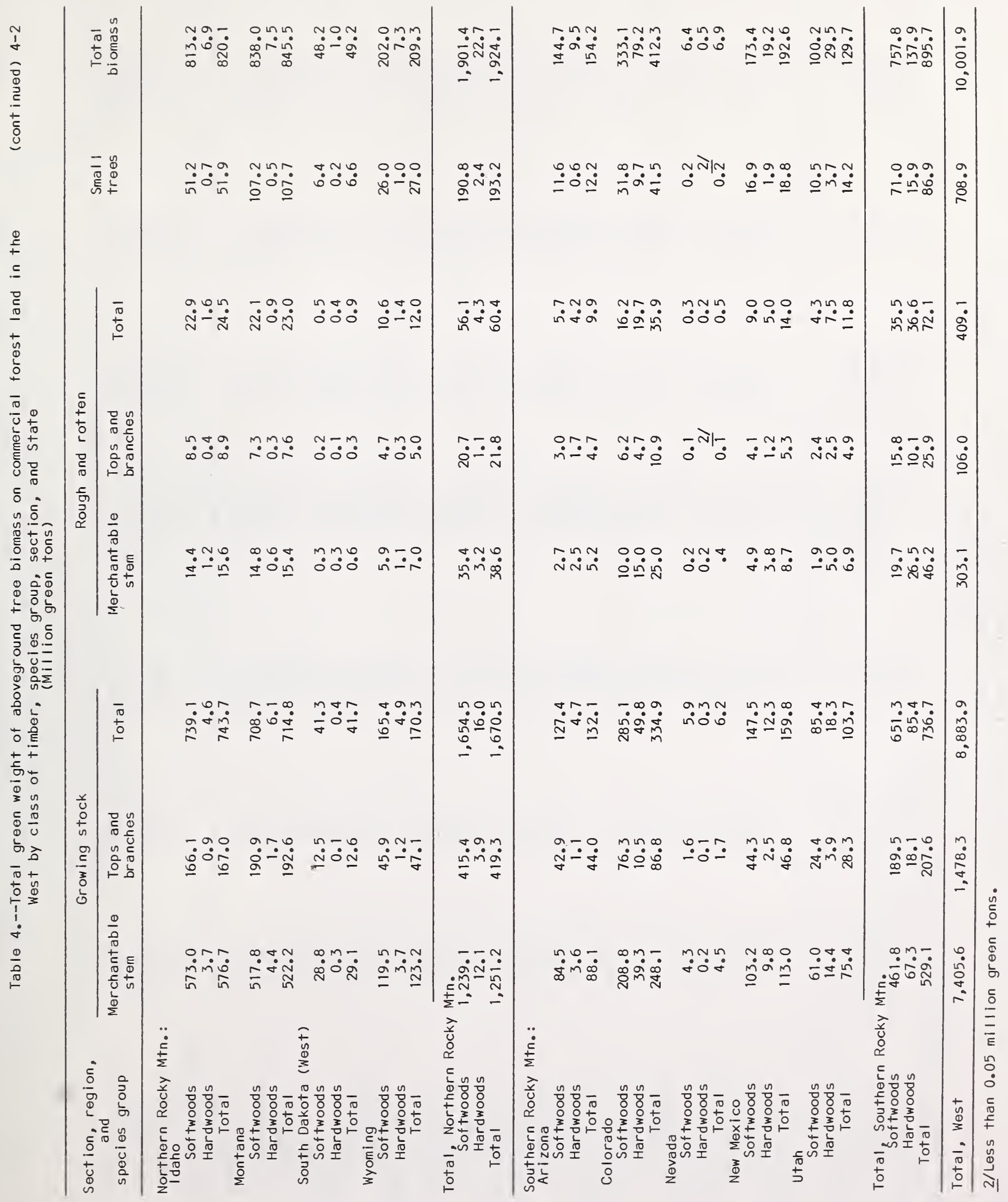




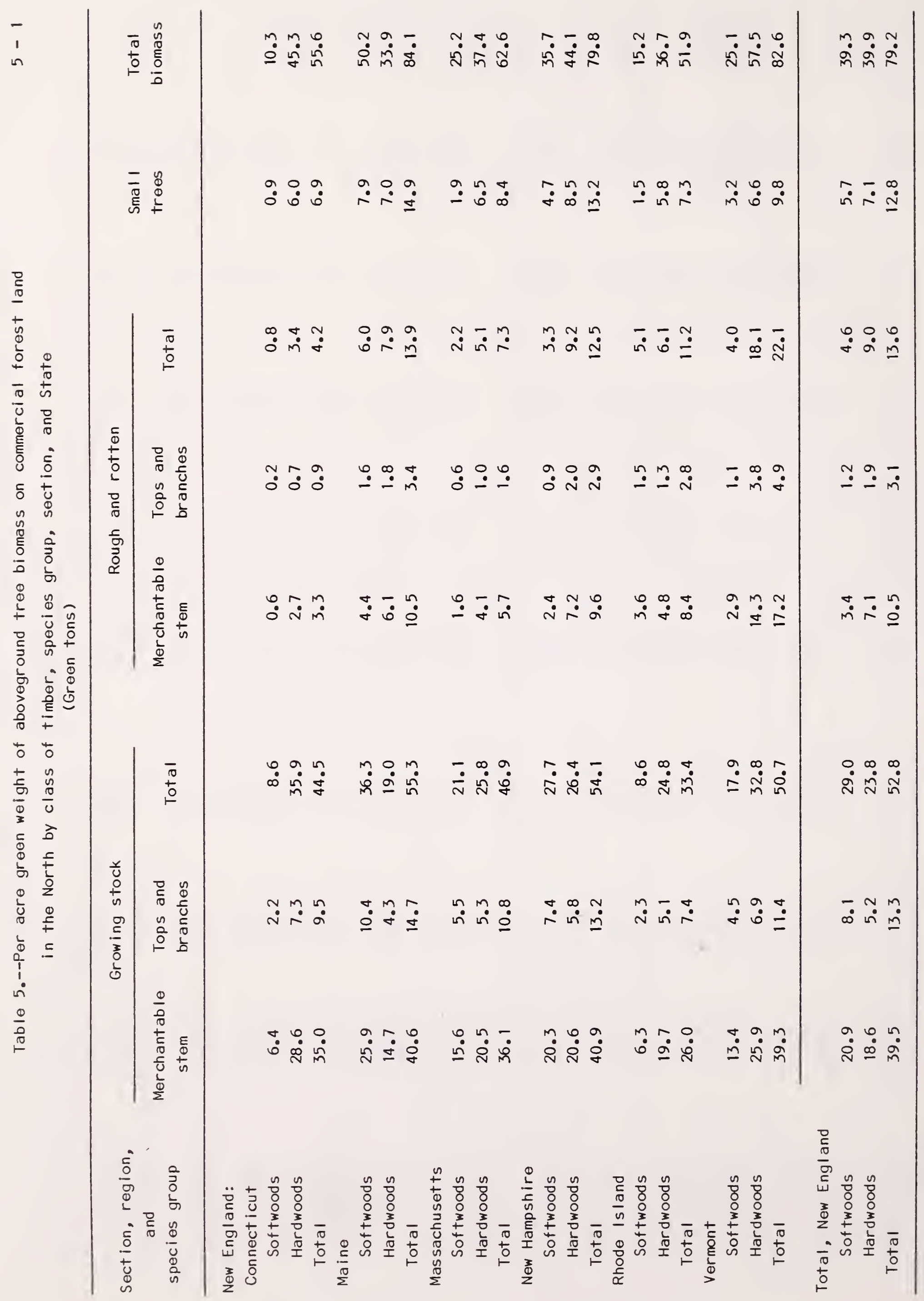




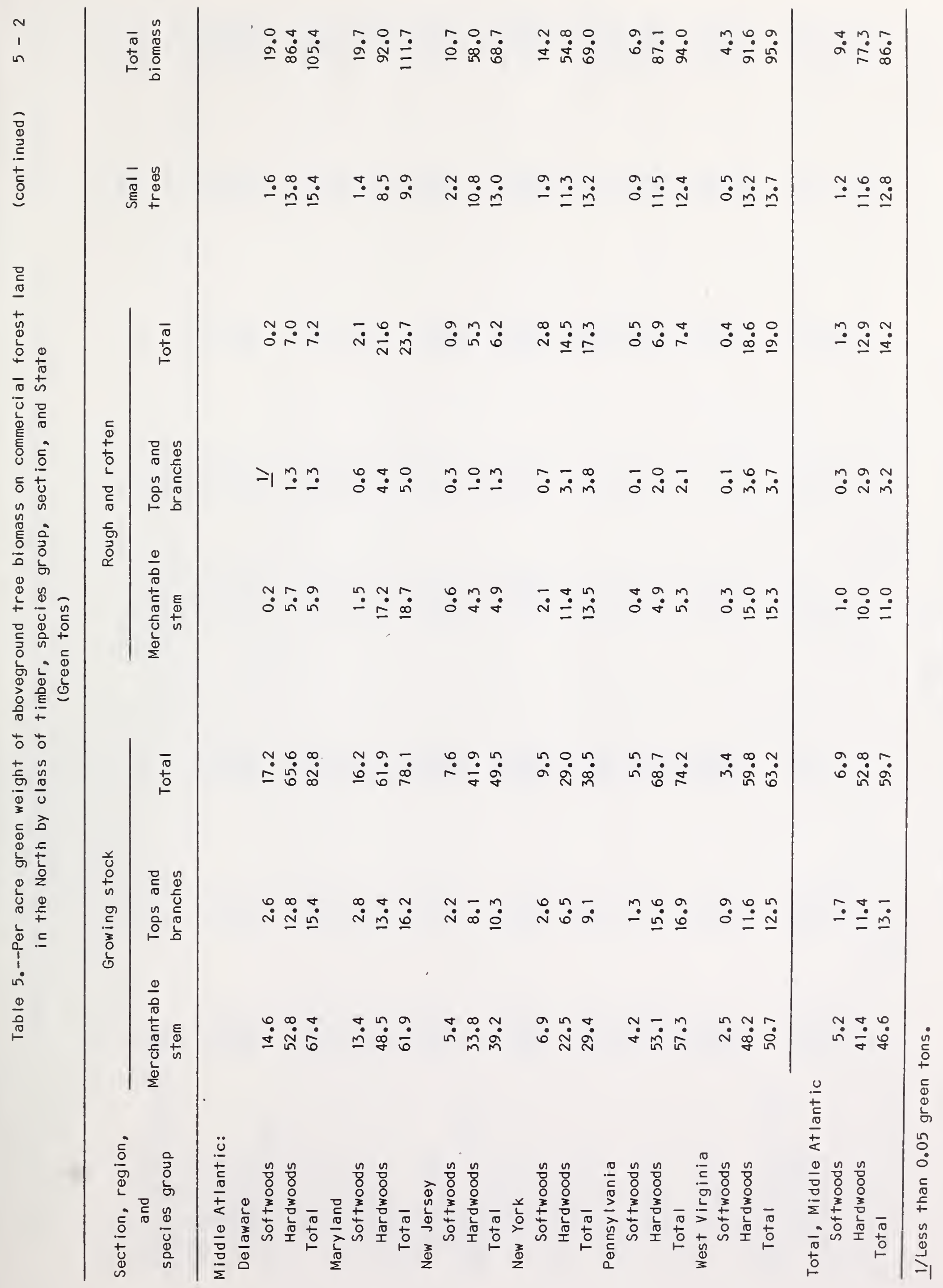




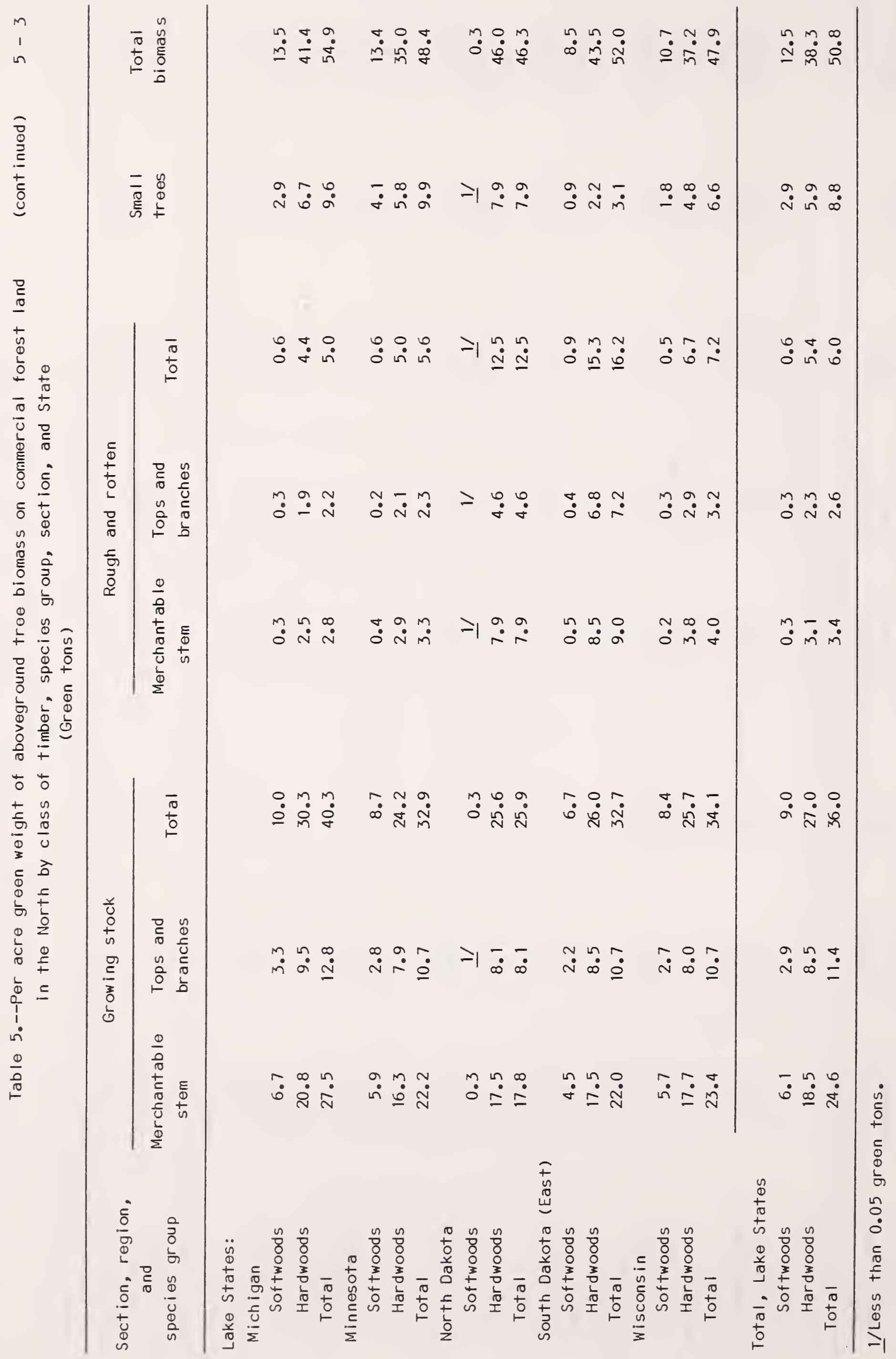




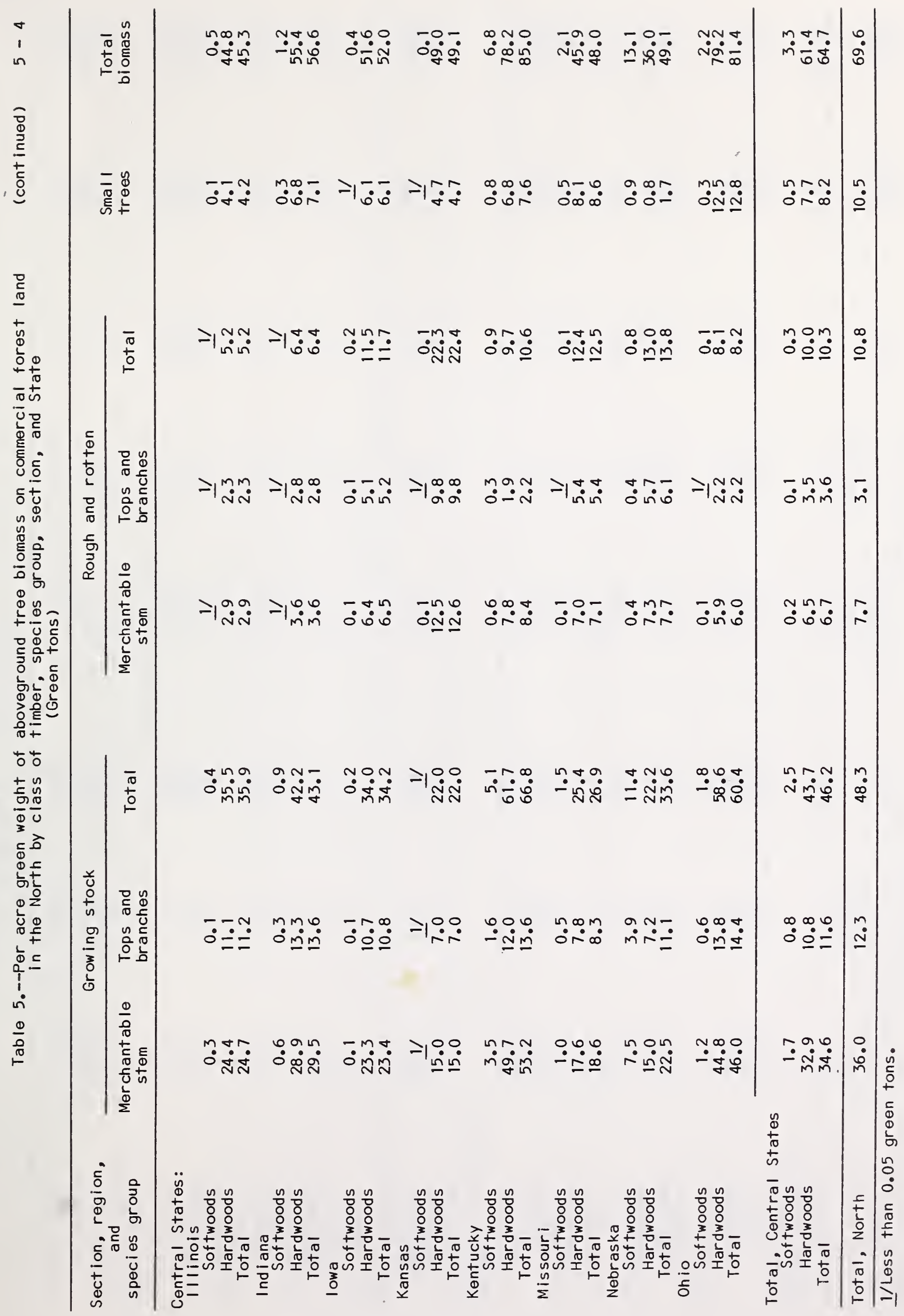




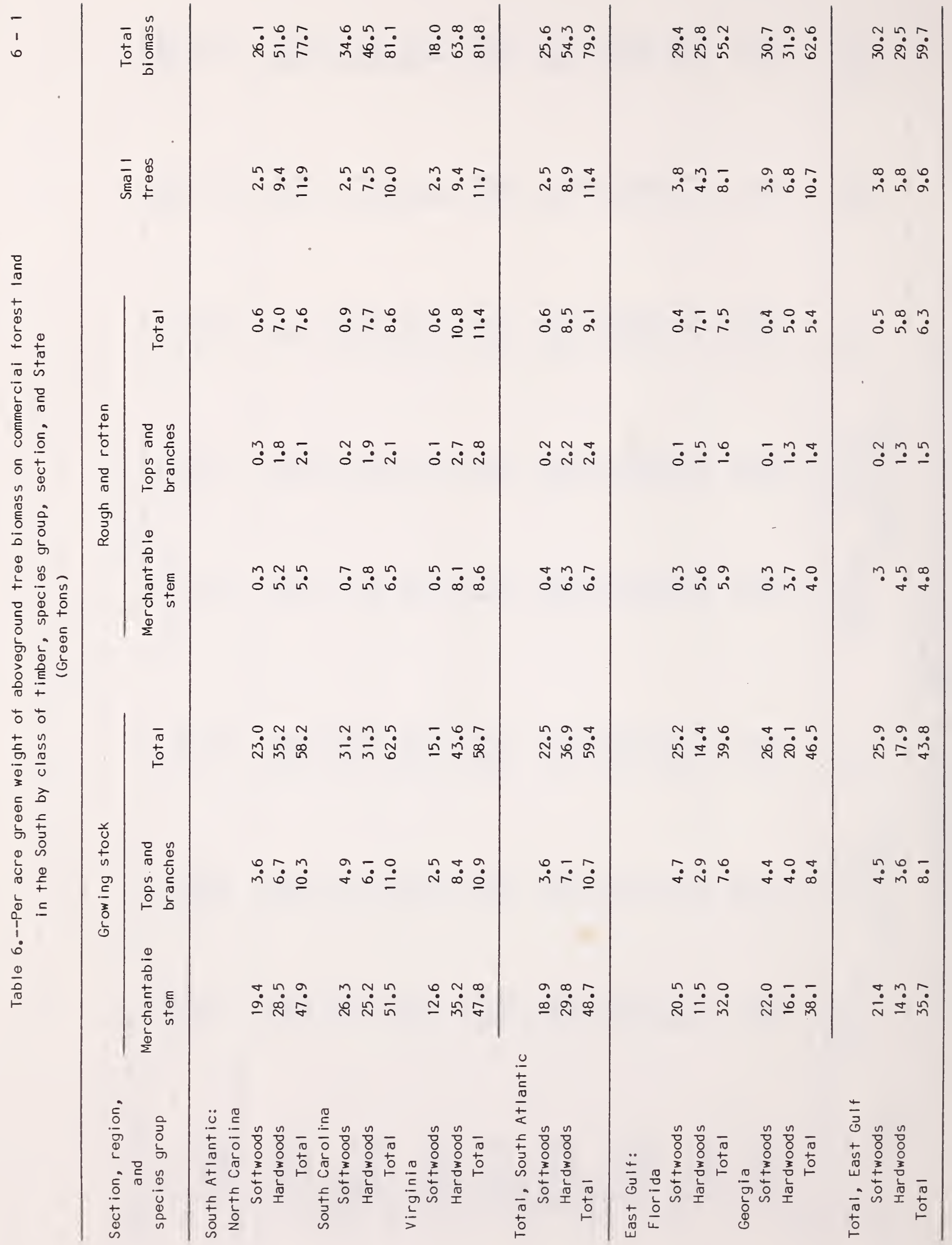




\begin{tabular}{|c|c|c|c|c|}
\hline 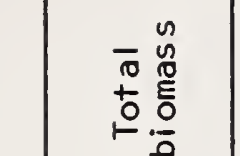 & 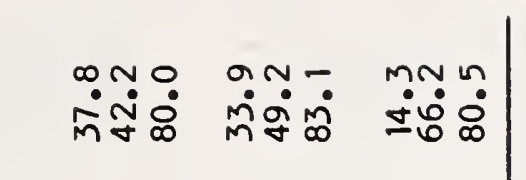 & 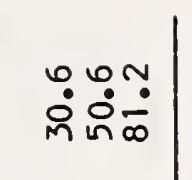 & 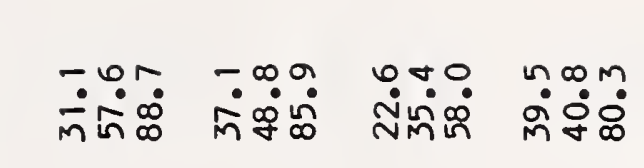 & 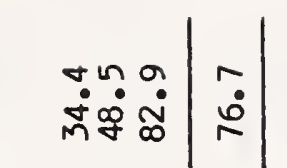 \\
\hline 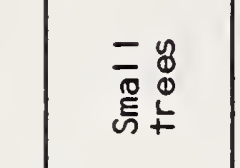 & 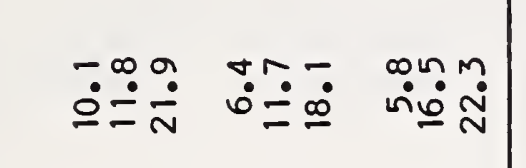 & 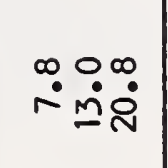 & 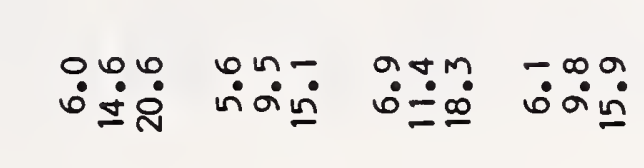 & 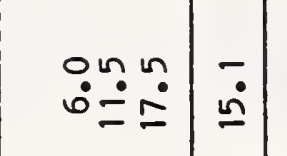 \\
\hline $\mid \frac{\bar{g}}{\dot{2}}$ & 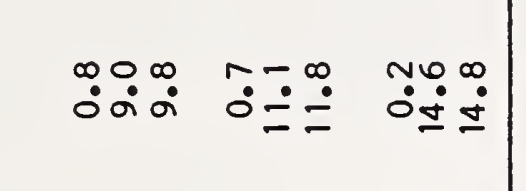 & 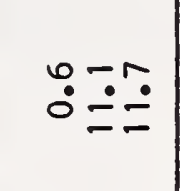 & 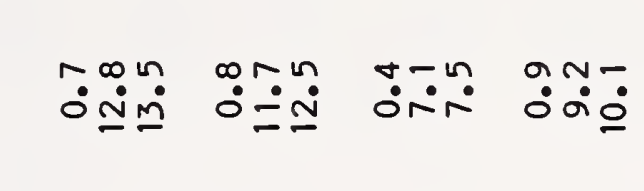 & 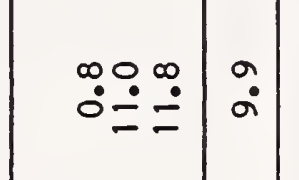 \\
\hline 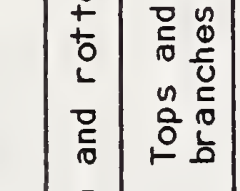 & 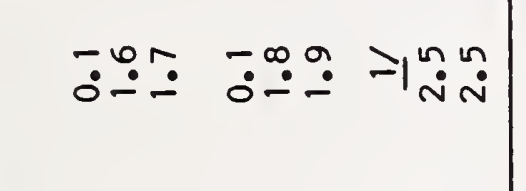 & 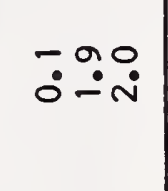 & 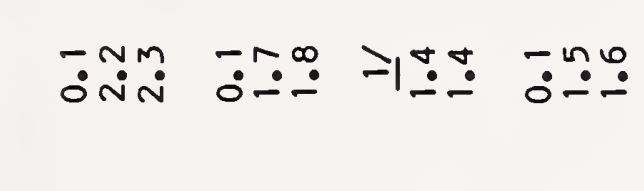 & 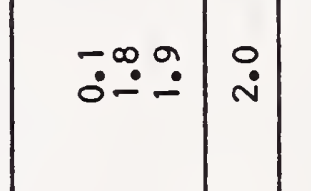 \\
\hline $\mid$ & 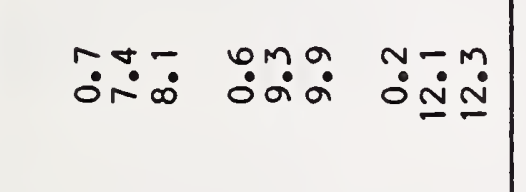 & 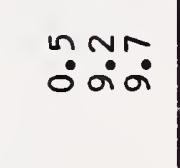 & 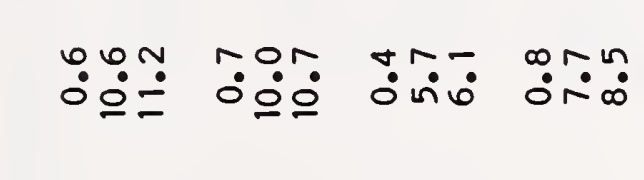 & \\
\hline$\frac{9}{2}$ & $\mid$ & 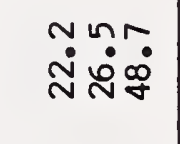 & 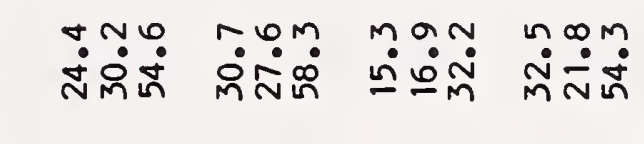 & 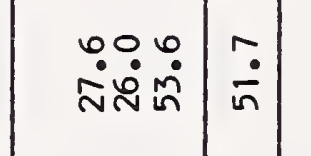 \\
\hline 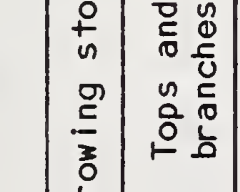 & 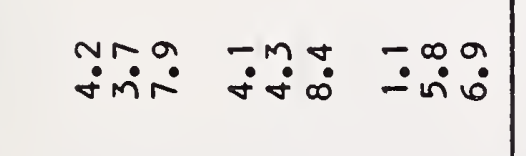 & 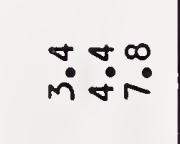 & 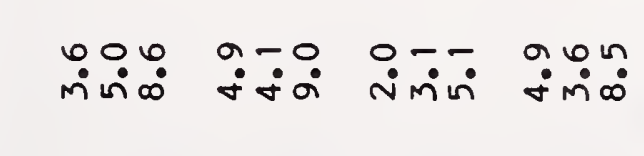 & 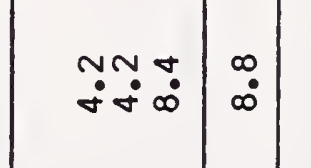 \\
\hline 类 & 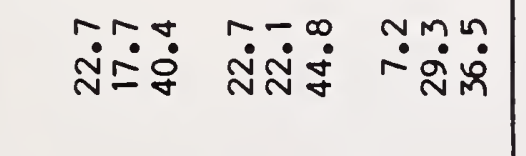 & 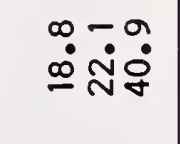 & 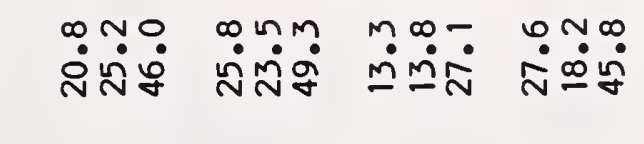 & 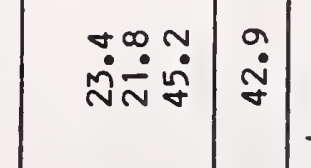 \\
\hline
\end{tabular}




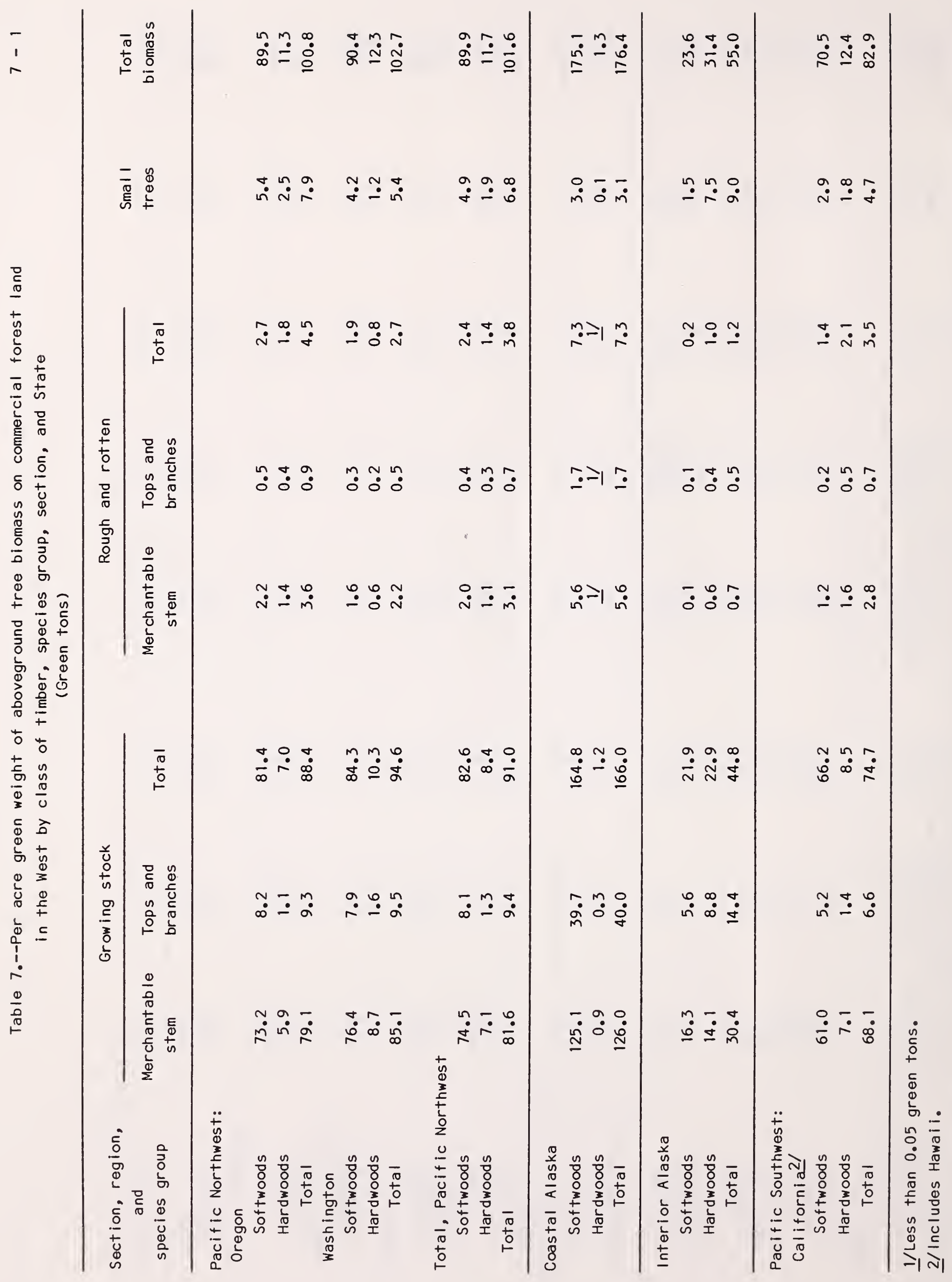




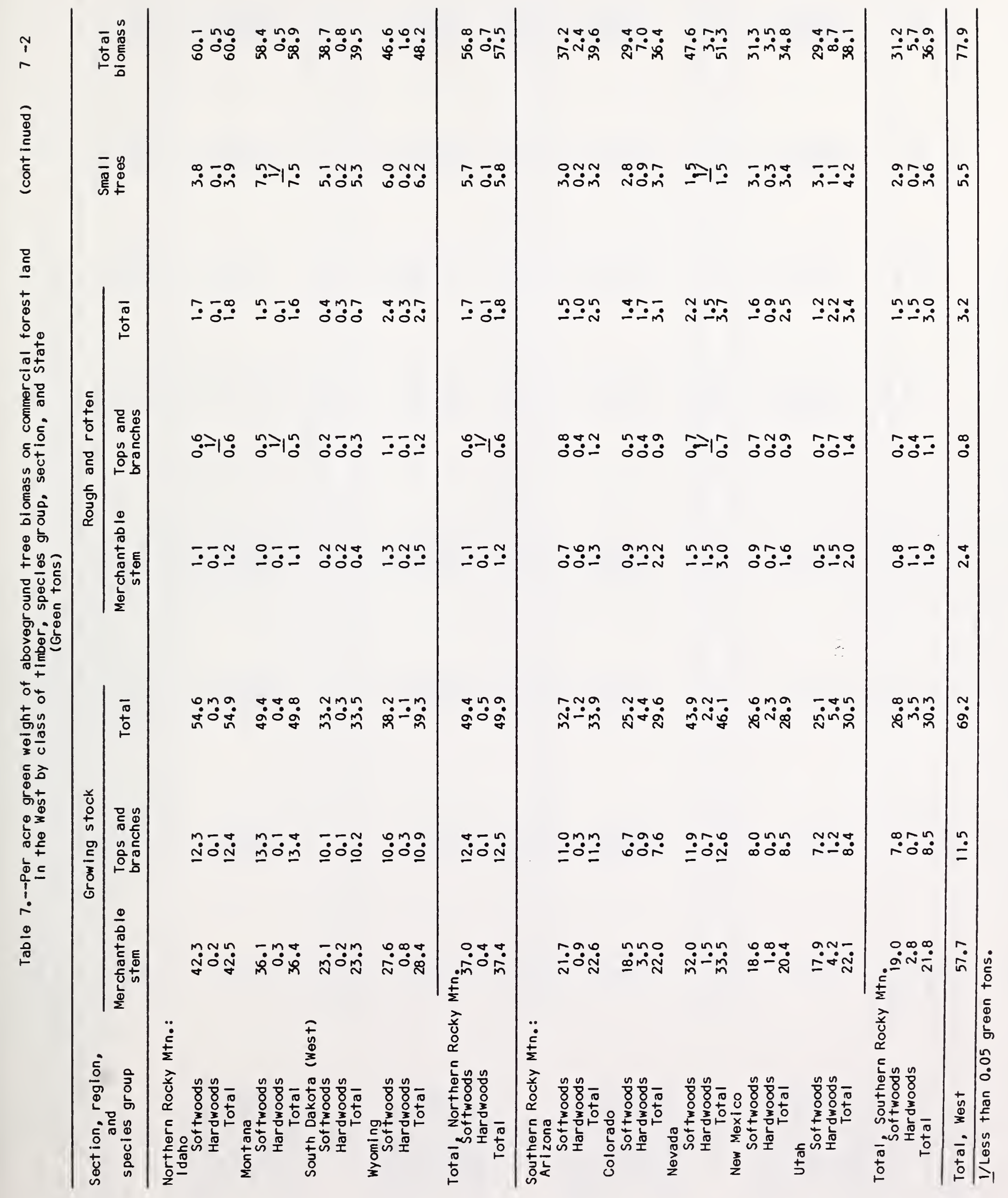




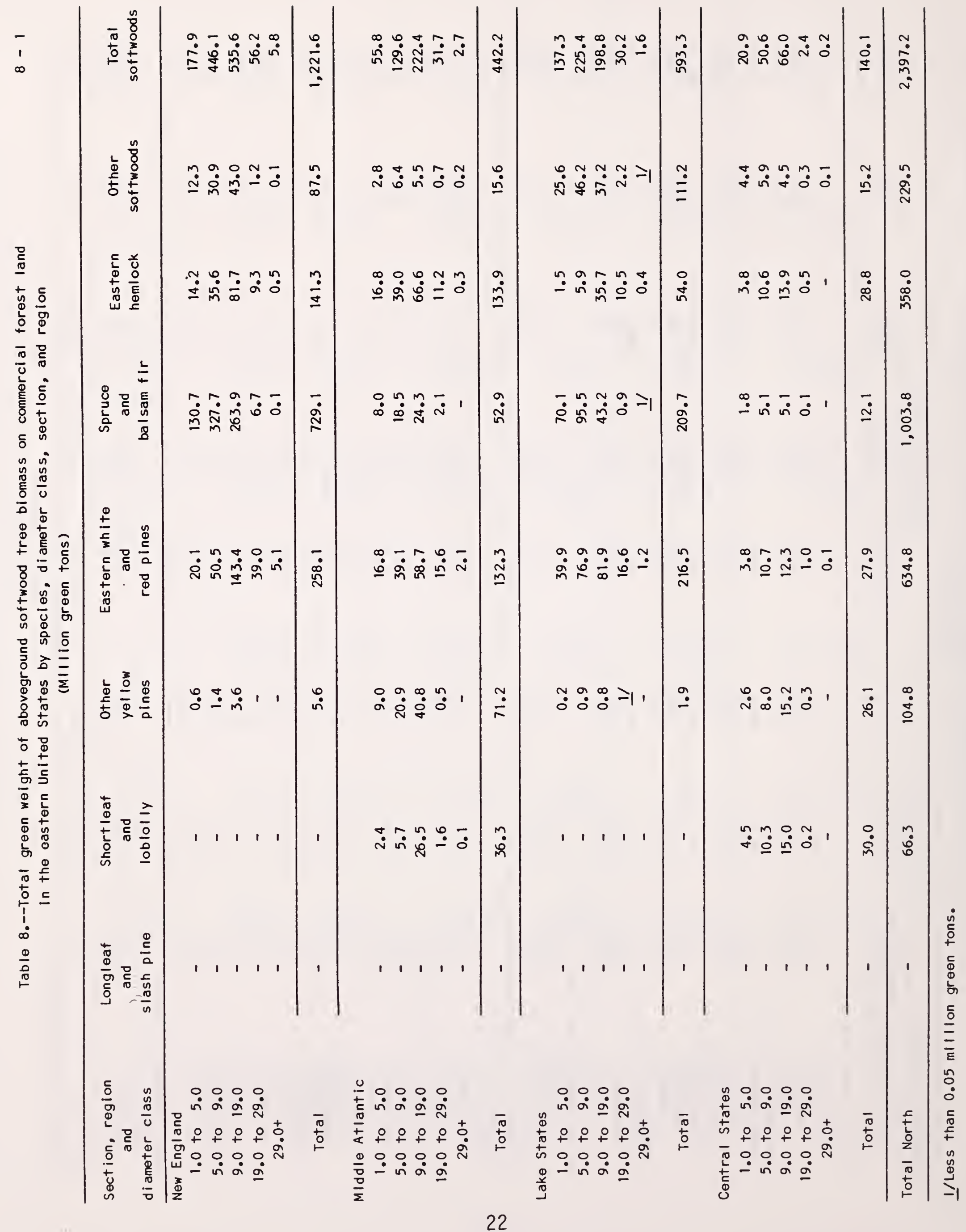




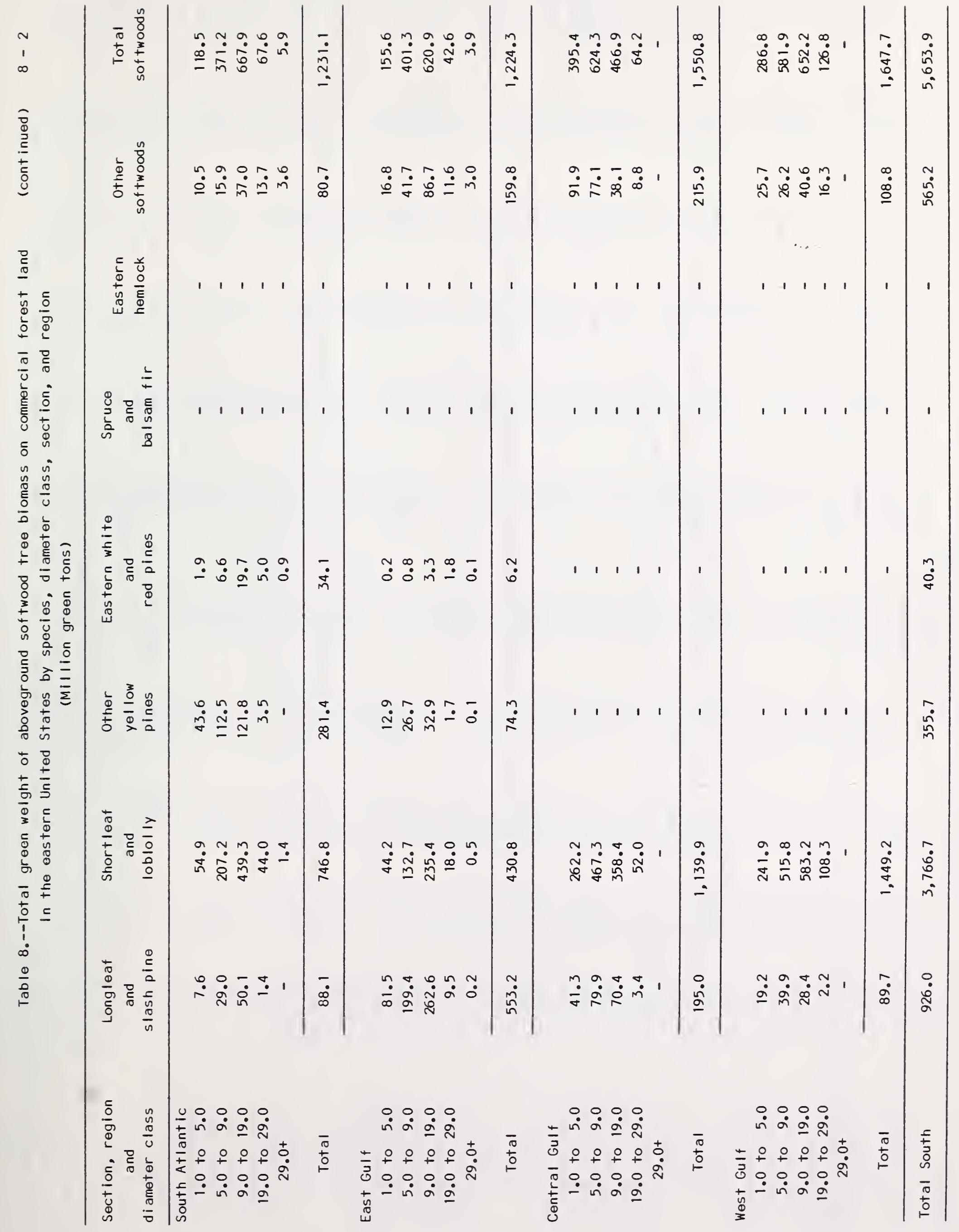




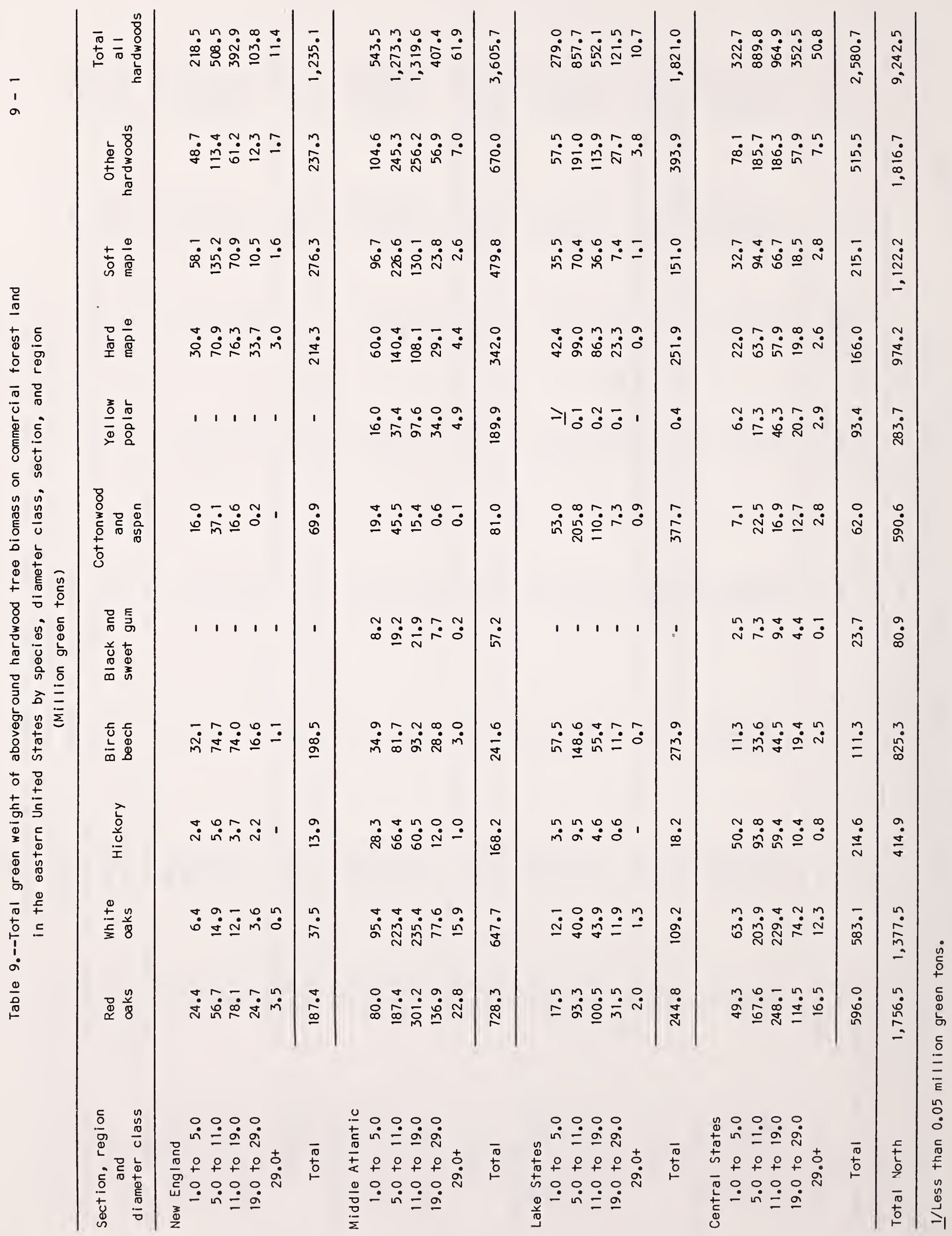




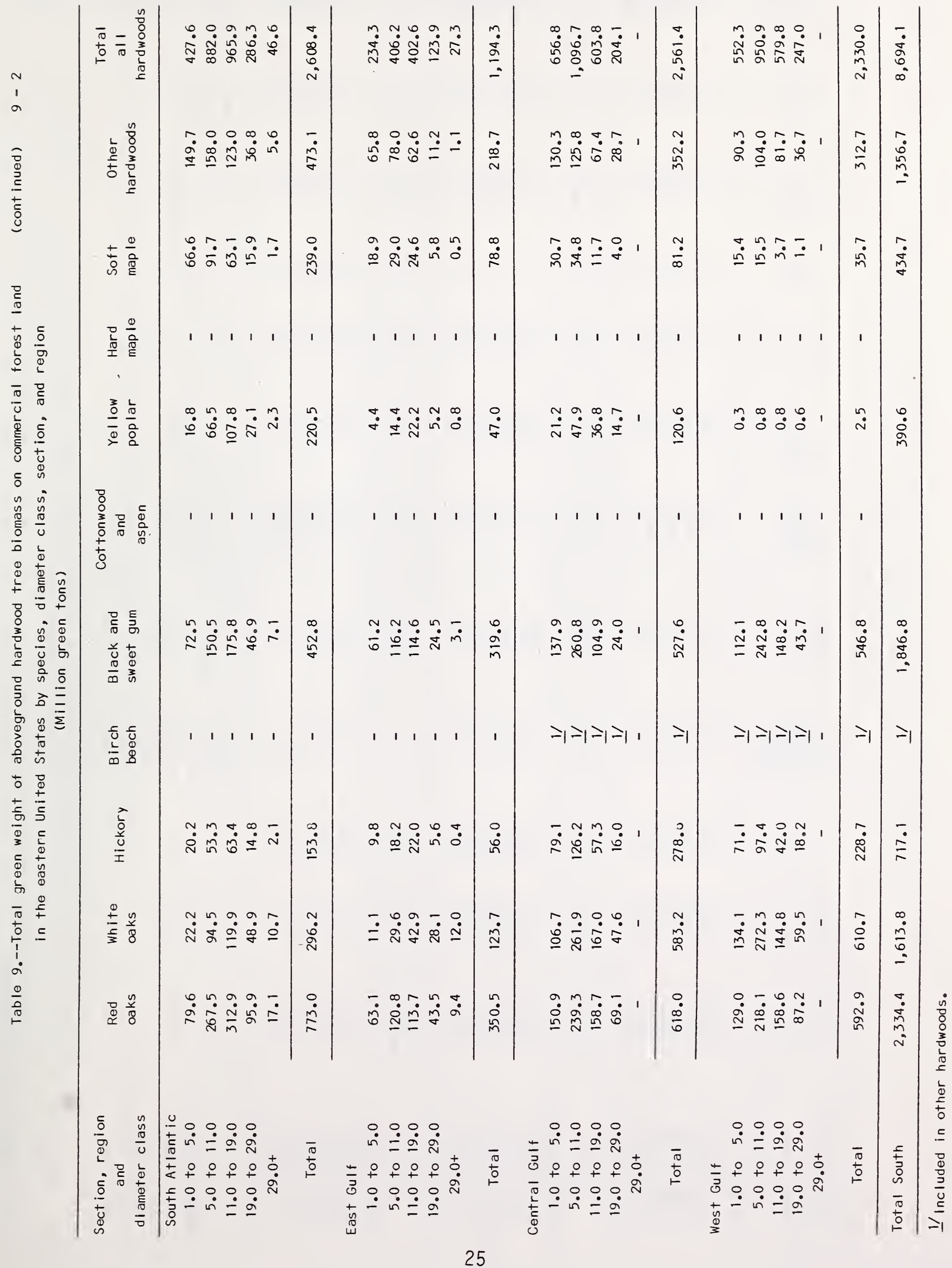




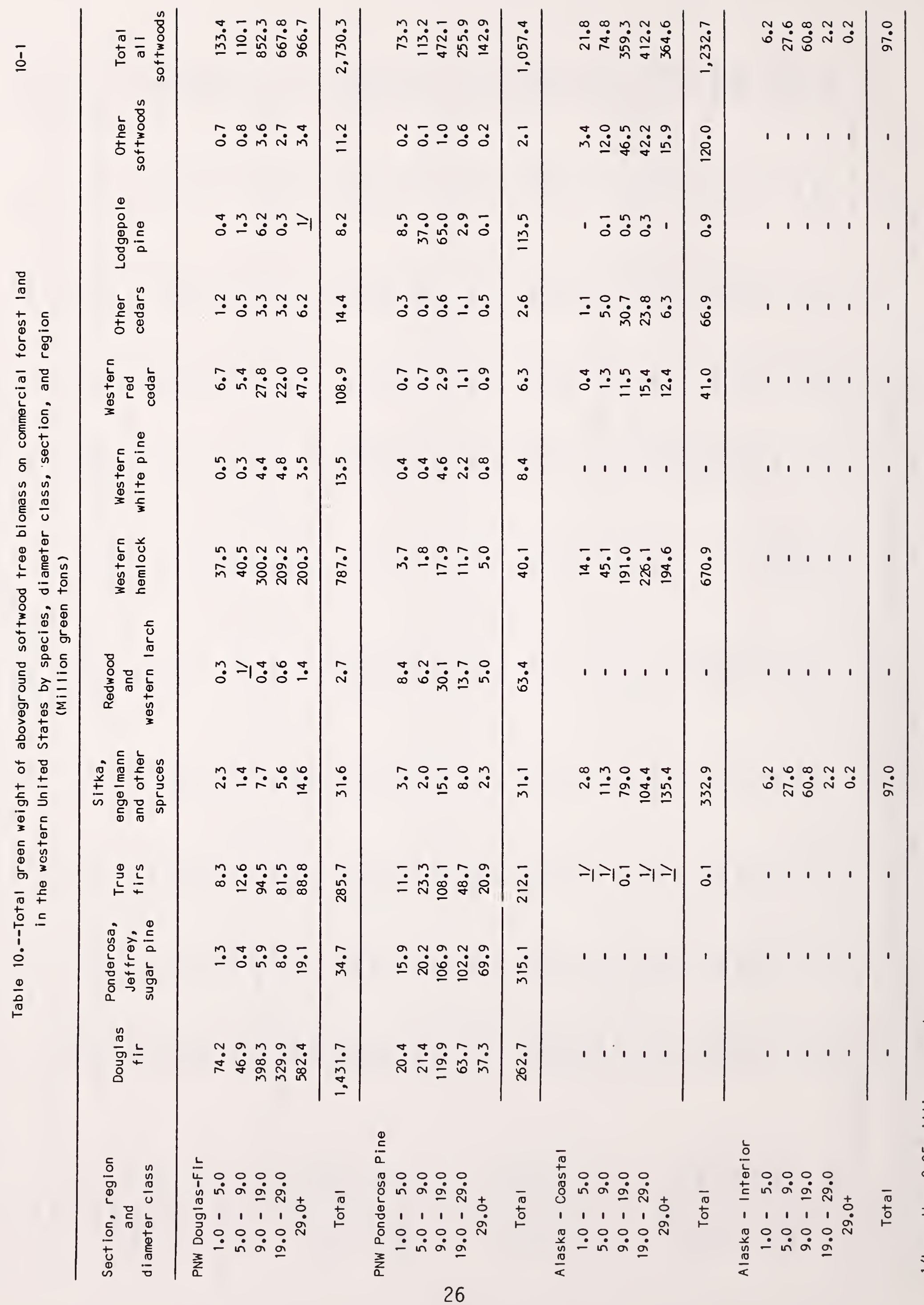

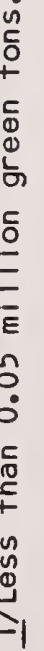




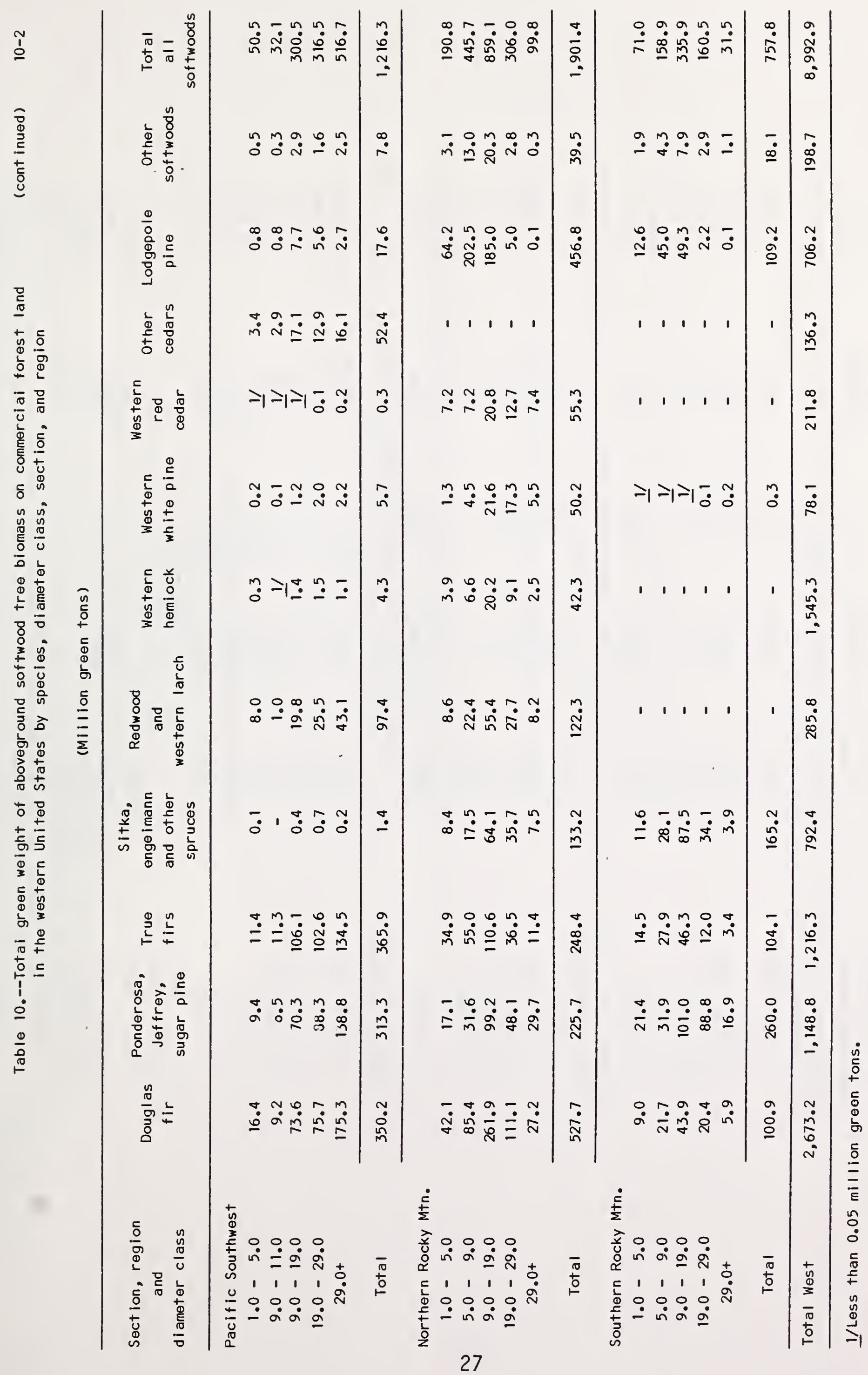




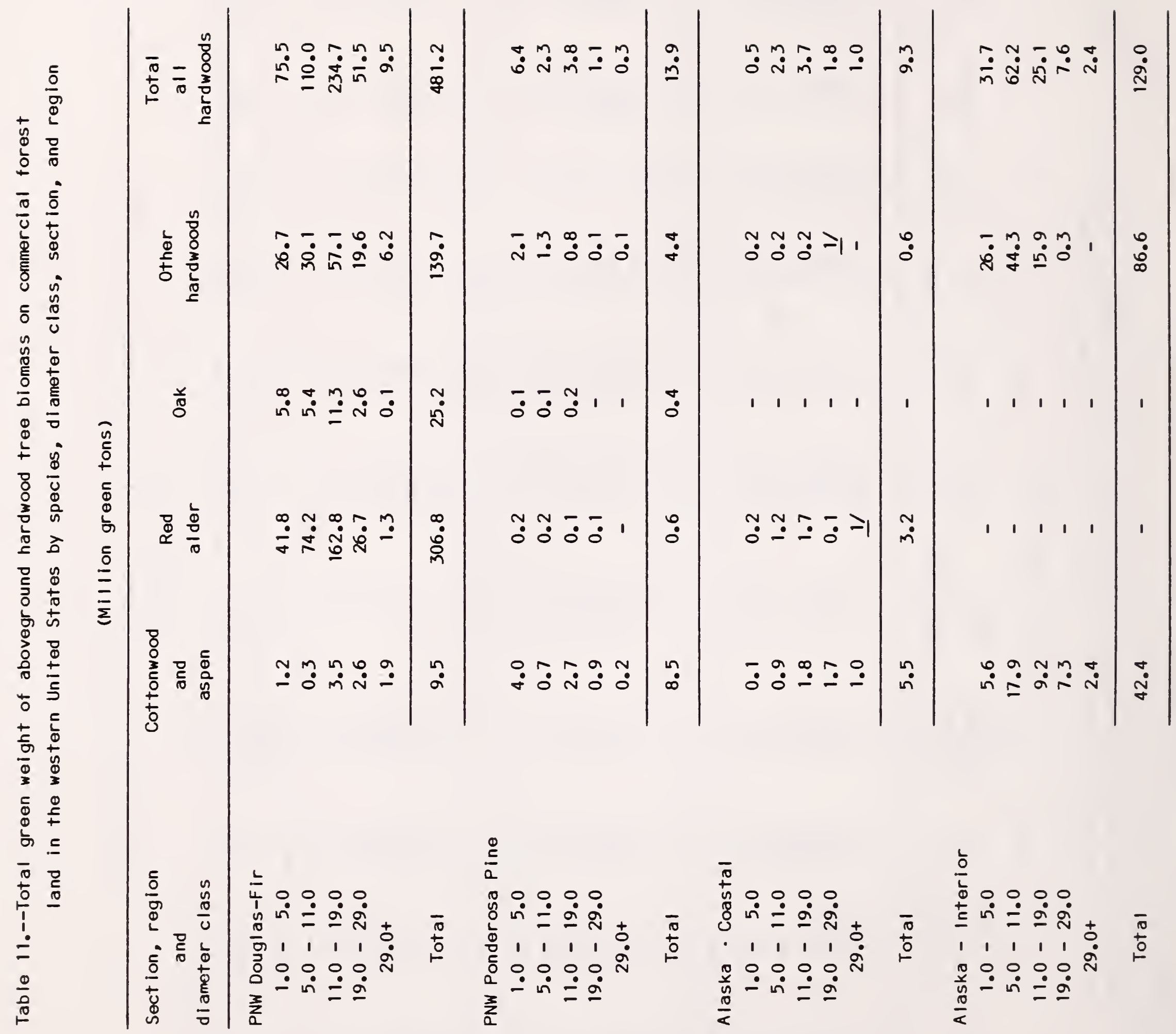




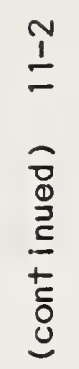

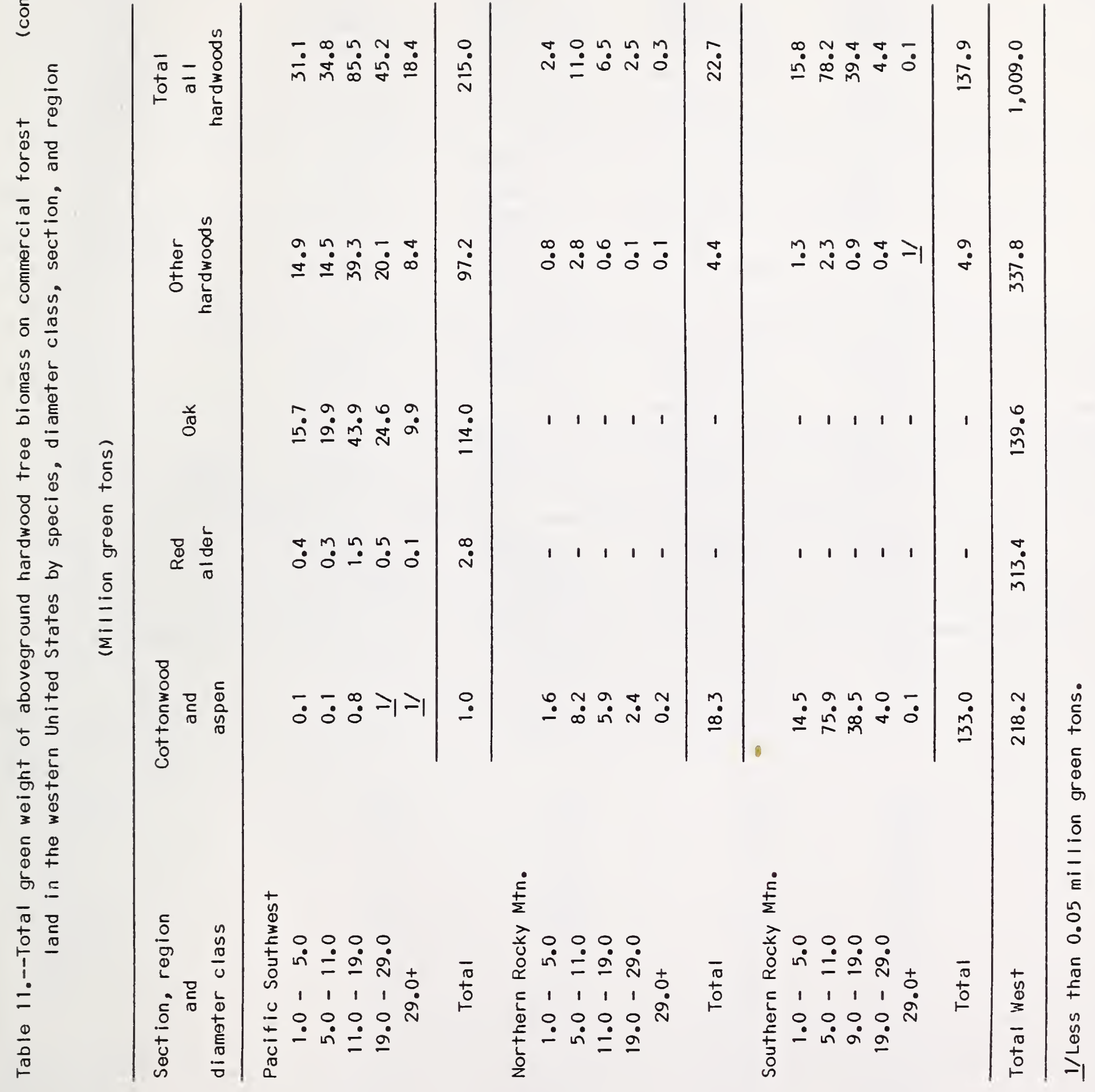


Table 12.--Total green weight of aboveground tree biomass on commercial forest land

in the United States by ownership, section, and region

(Million green tons)

\begin{tabular}{|c|c|c|c|c|c|}
\hline $\begin{array}{c}\text { Section and } \\
\text { region }\end{array}$ & $\begin{array}{c}\text { National } \\
\text { Forest }\end{array}$ & $\begin{array}{l}\text { Other } \\
\text { public }\end{array}$ & $\begin{array}{l}\text { Forest } \\
\text { industry }\end{array}$ & $\begin{array}{l}\text { Other } \\
\text { private }\end{array}$ & $\begin{array}{l}\text { Total, all } \\
\text { ownerships }\end{array}$ \\
\hline New England & 67.0 & 80.9 & 863.9 & $1,444.9$ & $2,456.7$ \\
\hline Middle Atlantic & 131.8 & 465.3 & 277.1 & $3,173.7$ & $4,047.9$ \\
\hline Lake States & 268.4 & 623.2 & 255.8 & $1,266.9$ & $2,414.3$ \\
\hline Central States & 165.9 & 99.4 & 50.3 & $2,405.2$ & $2,720.8$ \\
\hline Total, North & 633.1 & $1,268.8$ & $1,447.1$ & $8,290.7$ & $11,639.7$ \\
\hline South Atlantic & 299.7 & 136.7 & 427.7 & $2,975.4$ & $3,839.5$ \\
\hline East Gulf & 135.5 & 121.5 & 498.2 & $1,663.4$ & $2,418.6$ \\
\hline Central Gulf & 191.9 & 132.7 & 675.9 & $3,111.7$ & $4,112.2$ \\
\hline West Gulf & 317.2 & 124.4 & $1,059.0$ & $2,477.1$ & $3,977.7$ \\
\hline Total, South & 944.3 & 515.3 & $2,660.8$ & $10,227.6$ & $14,348.0$ \\
\hline Pacific Northwest & $2,074.3$ & 837.9 & 826.4 & 544.2 & $4,282.8$ \\
\hline Coastal Alaska & $1,151.5$ & 75.2 & 0.1 & 15.2 & $1,242.0$ \\
\hline Interior Alaska & - & 214.9 & - & 11.1 & 226.0 \\
\hline Pacific Southwest & 815.8 & 45.8 & 240.5 & 329.2 & $1,431.3$ \\
\hline Northern Rocky Mtn. & $1,244.6$ & 204.0 & 155.9 & 319.6 & $1,924.1$ \\
\hline Southern Rocky Mtn. & 587.0 & 131.7 & 1.0 & 176.0 & 895.7 \\
\hline Total, West & $5,873.2$ & $1,509.5$ & $1,223.9$ & $1,395.3$ & $10,001.9$ \\
\hline Total, All Regions & $7,450.6$ & $3,293.6$ & $5,331.8$ & $19,913.6$ & $35,989.6$ \\
\hline
\end{tabular}


Table 13.--Total green weight of aboveground tree biomass on commercial forest land in the North by ownership, section, region, and State

(Million green tons)

\begin{tabular}{|c|c|c|c|c|c|}
\hline $\begin{array}{l}\text { Section } \\
\text { and State }\end{array}$ & $\begin{array}{c}\text { National } \\
\text { Forest }\end{array}$ & $\begin{array}{l}\text { Other } \\
\text { public }\end{array}$ & $\begin{array}{l}\text { Forest } \\
\text { industry }\end{array}$ & $\begin{array}{l}\text { Other } \\
\text { private }\end{array}$ & $\begin{array}{l}\text { Total, all } \\
\text { ownerships }\end{array}$ \\
\hline \multicolumn{6}{|l|}{ New England: } \\
\hline Connecticut & - & 8.1 & - & 92.4 & 100.5 \\
\hline Maine & 3.0 & 22.9 & 728.4 & 666.0 & $1,420.3$ \\
\hline Massachusetts & - & 22.8 & 1.9 & 150.2 & 174.9 \\
\hline New Hampshire & 43.2 & 8.4 & 81.7 & 241.0 & 374.3 \\
\hline Rhode Island & - & 1.6 & - & 18.9 & 20.5 \\
\hline Vermont & 20.8 & 17.1 & 51.9 & 276.4 & 366.2 \\
\hline Total, New England & 67.0 & 80.9 & 863.9 & $1,444.9$ & $2,456.7$ \\
\hline \multicolumn{6}{|l|}{ Middle Atlantic: } \\
\hline Delaware & - & 1.5 & 3.1 & 35.9 & 40.5 \\
\hline Maryland & - & 26.3 & 16.1 & 239.2 & 281.6 \\
\hline New Jersey & - & 21.9 & 1.1 & 104.6 & 127.6 \\
\hline New York & - & 63.7 & 81.6 & 855.4 & $1,000.7$ \\
\hline Pennsy Ivania & 47.6 & 326.4 & 88.6 & $1,034.1$ & $1,496.7$ \\
\hline West Virginia & 84.2 & 25.5 & 86.6 & 904.5 & $1,100.8$ \\
\hline Total, Middle Atlantic & 131.8 & 465.3 & 277.1 & $3,173.7$ & $4,047.9$ \\
\hline \multicolumn{6}{|l|}{ Lake States: } \\
\hline Michigan & 114.9 & 212.5 & 139.2 & 563.8 & $1,030.4$ \\
\hline Minnesota & 84.3 & 267.6 & 37.4 & 273.0 & 662.3 \\
\hline North Dakota & 0.1 & 1.4 & - & 14.4 & 15.9 \\
\hline South Dakota (East) & - & 3.4 & - & 8.2 & 11.6 \\
\hline Wiscons in & 69.1 & 138.3 & 79.2 & 407.5 & 694.1 \\
\hline Total, Lake States & 268.4 & 623.2 & 255.8 & $1,266.9$ & $2,414.3$ \\
\hline \multicolumn{6}{|l|}{ Central: } \\
\hline Illinois & 9.0 & 3.2 & 0.6 & 154.6 & 167.4 \\
\hline Indiana & 9.2 & 14.9 & 1.2 & 190.5 & 215.8 \\
\hline lowa & - & 8.2 & 0.9 & 66.9 & 76.0 \\
\hline Kansas & - & 2.3 & - & 55.9 & 58.2 \\
\hline Kentucky & 56.1 & 26.0 & 21.8 & 907.7 & $1,011.6$ \\
\hline Missouri & 76.3 & 16.5 & 17.8 & 479.5 & 590.1 \\
\hline Nebraska & 2.5 & 1.2 & - & 35.0 & 38.7 \\
\hline Onio & 12.8 & 27.1 & 8.0 & 515.1 & 563.0 \\
\hline Total, Central & 165.9 & 99.4 & 50.3 & $2,405.2$ & $2,720.8$ \\
\hline Total, North & 633.1 & $1,268.8$ & $1,447.1$ & $8,290.7$ & $11,639.7$ \\
\hline
\end{tabular}


Table 14.--Total green weight of aboveground tree biomass on commercial forest land

in the South by ownership, section, region, and State

(Million green tons)

\begin{tabular}{|c|c|c|c|c|c|}
\hline $\begin{array}{l}\text { Section } \\
\text { and State }\end{array}$ & $\begin{array}{c}\text { National } \\
\text { Forest }\end{array}$ & $\begin{array}{l}\text { Other } \\
\text { public }\end{array}$ & $\begin{array}{l}\text { Forest } \\
\text { industry }\end{array}$ & $\begin{array}{l}\text { Other } \\
\text { private }\end{array}$ & $\begin{array}{l}\text { Total, all } \\
\text { ownerships }\end{array}$ \\
\hline \multicolumn{6}{|l|}{ South AtIantic: } \\
\hline North Carolina & 102.5 & 40.6 & 136.9 & $1,238.6$ & $1,518.6$ \\
\hline South Carolina & 60.9 & 43.4 & 172.2 & 737.5 & $1,014.0$ \\
\hline Virginia & 136.3 & 52.7 & 118.6 & 999.3 & $1,306.9$ \\
\hline Total, South Atlantic & 299.7 & 136.7 & 427.7 & $2,975.4$ & $3,839.5$ \\
\hline \multicolumn{6}{|l|}{$\overline{\text { East Gulf: }}$} \\
\hline Florida & 60.8 & 67.0 & 237.1 & 499.1 & 864.0 \\
\hline Georgia & 74.7 & 54.5 & 261.1 & $1,164.3$ & $1,554.6$ \\
\hline Total, East Gulf & 135.5 & 121.5 & 498.2 & $1,663.4$ & $2,418.6$ \\
\hline \multicolumn{6}{|l|}{ Central Gulf: } \\
\hline Alabama & 50.3 & 31.3 & 336.3 & $1,288.4$ & $1,706.3$ \\
\hline Mississippi & 93.3 & 46.1 & 249.2 & 984.1 & $1,372.7$ \\
\hline Tennessee & 48.3 & 55.3 & 90.4 & 839.2 & $1,033.2$ \\
\hline Total, Central Gulf & 191.9 & 132.7 & 675.9 & $3,111.7$ & $4,112.2$ \\
\hline \multicolumn{6}{|l|}{ West Gulf: } \\
\hline Arkansas & 208.4 & 50.4 & 375.4 & 839.4 & $1,473.6$ \\
\hline Louisiana & 49.8 & 38.0 & 323.2 & 837.2 & $1,248.2$ \\
\hline Oklahoma & 12.7 & 19.9 & 57.5 & 160.7 & 250.8 \\
\hline Texas & 46.3 & 16.1 & 302.9 & 639.8 & $1,005.1$ \\
\hline Total, West Gulf & 317.2 & 124.4 & $1,059.0$ & $2,477.1$ & $3,977.7$ \\
\hline Total, South & 944.3 & 515.3 & $2,660.8$ & $10,227.6$ & $14,348.0$ \\
\hline
\end{tabular}


Table 15.--Total green weight of aboveground tree biomass on commercial forest land in the West by ownership, section, region, and State

(Million green tons)

\begin{tabular}{|c|c|c|c|c|c|}
\hline $\begin{array}{c}\text { Section } \\
\text { and State }\end{array}$ & $\begin{array}{c}\text { National } \\
\text { Forest }\end{array}$ & $\begin{array}{l}\text { Other } \\
\text { public }\end{array}$ & $\begin{array}{l}\text { Forest } \\
\text { industry }\end{array}$ & $\begin{array}{c}\text { Other } \\
\text { private }\end{array}$ & $\begin{array}{l}\text { Total, all } \\
\text { ownerships }\end{array}$ \\
\hline \multicolumn{6}{|l|}{ Pacific Northwest: } \\
\hline Oregon & $1,396.8$ & 427.4 & 373.6 & 244.2 & $2,442.0$ \\
\hline Washington & 677.5 & 410.5 & 452.8 & 300.0 & $1,840.8$ \\
\hline Total, Pacific Northwest & $2,074.3$ & 837.9 & 826.4 & 544.2 & $4,282.8$ \\
\hline Coastal Alaska & $1,151.5$ & 75.2 & 0.1 & 15.2 & $1,242.0$ \\
\hline Interior Alaska & - & 214.9 & - & 11.1 & 226.0 \\
\hline \multicolumn{6}{|l|}{ Pacific Southwest } \\
\hline Californiall & 815.8 & 45.8 & 240.5 & 329.2 & $1,431.3$ \\
\hline \multicolumn{6}{|l|}{ Northern Rocky Mtn.: } \\
\hline Idaho & 540.1 & 90.7 & 80.4 & 108.9 & 820.1 \\
\hline Montana & 506.3 & 89.9 & 72.7 & 176.6 & 845.5 \\
\hline South Dakota (West) & 39.1 & 2.8 & 0.7 & 6.6 & 49.2 \\
\hline Wyoming & 159.1 & 20.6 & 2.1 & 27.5 & 209.3 \\
\hline Total, Northern Rocky M+n. & $1,244.6$ & 204.0 & 155.9 & 319.6 & $1,924.1$ \\
\hline \multicolumn{6}{|l|}{ Southern Rocky $M+n_{.}$: } \\
\hline Arizona & 101.6 & 47.5 & - & 5.1 & 154.2 \\
\hline Colorado & 295.3 & 25.6 & 0.6 & 90.8 & 412.3 \\
\hline Nevada & 2.8 & 0.2 & 0.4 & 3.5 & 6.9 \\
\hline New Mexico & 95.5 & 40.8 & - & 56.3 & 192.6 \\
\hline Utah & 91.8 & 17.6 & - & 20.3 & 129.7 \\
\hline Total Southern Rocky M+n. & 587.0 & 131.7 & 1.0 & 176.0 & 895.7 \\
\hline Total, West & $5,873.2$ & $1,509.5$ & $1,223.9$ & $1,395.3$ & $10,001.9$ \\
\hline
\end{tabular}

1/ Includes Hawai i 
Tree biomass - the green weight of the wood and bark above the 1-foot stump.

Merchantable stem - the main stem of the tree between the 1-foot stump and the 4-inch top; wood and bark.

Tree crown (top and branches) - the main stem above the 4-inch top plus branches, excluding the foliage.

Growing stock trees - all live trees of commercial species, 5.0 inches dbh or larger, except rough and rotten trees.

Rotten trees - live trees of commercial species that do not contain a sawlog, now or prospectively, primarily because of rot, (e.g. when rot accounts for more than 50 percent of the total cull volume).

Rough trees - (a) live trees of commercial species that do not contain a sawlog, now or prospectively, primarily because of roughness, poor form, splits, and cracks, and with less than one-third of the gross tree volume in sound material; and (b) all live trees of noncommercial species.

Small trees - live trees that are less than 5.0 inches dbh.
Commercial forest 1 and - forest

land producing or capable of producing annual crops of industrial wood at not less than 20 cubic feet per acre and that is not withdrawn from timber utilization.

Industrial wood - All commercial roundwood products except fuelwood.

Commercial species - tree species suitable for industrial wood products.

Other public lands - 1 ands administered by such federal agencies as the Bureau of Indian Affairs and Bureau of Land Management and those administered by State, county, and municipal organizations.

Forest industry lands - Lands owned by companies or individuals operating wood-using plants.

Other private lands - all private ownerships except forest industry lands.

Softwoods - coniferous trees, usually evergreen, and having needles or scale-like leaves.

Hardwoods - dicotyledonous trees, usually broad-leaved and deciduous. 

